\title{
Netrin-1 Ameliorates Postoperative Delirium-like Behavior in Aged Mice by Suppressing Neuroinflammation and Restoring Impaired Blood Brain Barrier Permeability
}

Ke Li

Wuhan University Zhongnan Hospital

Jiayu Wang

Wuhan University Zhongnan Hospital

\section{Lei Chen}

Wuhan University Zhongnan Hospital

Meimei Guo

Wuhan University Zhongnan Hospital

Ying Zhou

Wuhan University Zhongnan Hospital

Xiaofeng Li

Wuhan University Zhongnan Hospital

Zongze Zhang

Wuhan University Zhongnan Hospital

Mian Peng ( $\sim$ Mianpeng@whu.edu.cn )

Zhongnan Hospital Of Wuhan University

\section{Research}

Keywords: postoperative delirium, neuroinflammation, brain blood barrier, microglia cell, Netrin-1

Posted Date: November 18th, 2020

DOI: https://doi.org/10.21203/rs.3.rs-109643/v1

License: (c) (i) This work is licensed under a Creative Commons Attribution 4.0 International License. Read Full License

Version of Record: A version of this preprint was published at Frontiers in Molecular Neuroscience on January 14th, 2022. See the published version at https://doi.org/10.3389/fnmol.2021.751570. 


\section{Abstract}

Background Postoperative delirium (POD) is a common and serious postoperative complication in elderly patients, of which the underlying mechanism is elusive and without effective therapy at present. In recent years, the neuroinflammatory hypothesis has been developed in the pathogenesis of POD. Netrin-1, an axonal guidance molecule, has been reported to have strong inflammatory regulatory and neuroprotective effects.

Methods We applied treatment with Netrin- $1(45 \mu \mathrm{g} / \mathrm{kg})$ in aged mice by using the POD model with a simple laparotomy to assess systemic inflammatory, neuroinflammation by detecting interleukin-6 (IL-6), interleukin-10 (IL-10), high mobility group box chromosomal protein-1(HMGB-1) and assessing the reactive states of microglia, permeability of blood-brain barrier (BBB) by detecting cell junction proteins and leakage of dextran, and behavior of the aged mice.

Results We found that a single dose of Netrin-1 prophylaxis decreased the expression of IL- 6 and HMGB1 , and upregulated the expression of IL-10 in peripheral blood, hippocampus and prefrontal cortex. Nerin1 reduced activation of microglia cells in the hippocampus and prefrontal cortex and improved the PODlike behavior. Besides, Netrin-1 also attenuated the anesthesia/surgery-induced increase in BBB permeability by up-regulating the expression of tight junction-associated proteins such as ZO-1, claudin-5, and occludin.

Conclusions These findings confirm the anti-inflammatory and BBB protective effects of Netrin-1 in an inflammatory environment in vivo and provide better insights into the pathophysiology and potential treatment of POD.

\section{Background}

Postoperative delirium (POD) is a state of acute cerebral dysfunction characterized by fluctuating and concurrent disturbances of attention, cognition, psychomotor behavior, emotion and sleep-wake rhythm[1]. It is a common complication occurring mainly within 1 week after surgery and anesthesia [2]. POD may lead to greater length of hospital stay, increased hospitalization costs, decreased life independence, increased morbidity and mortality, and has potential to induce long-term cognitive dysfunction, and even dementia[3, 4]. Advanced age was reported to be an independent risk factor for the development of POD $[2,5]$. With the increasing aging of the population in global, the number of elderly people who need surgery/anesthesia treatment has been increasing, as well as the incidence of POD. Unfortunately, there are no effective therapies for this complication for the undefined underlying pathophysiology.

In recent years, more and more studies have shown that the occurrence of POD is closely related to neuroinflammation $[4,6,7]$. Aseptic surgical trauma provokes a homeostatic neuroinflammatory response, which when dysregulated, harmful consequences can follow. Surgery can result in an elevated level of proinflammatory cytokines such as tumor necrosis factor-a (TNF-a) and interleukin-6 (IL-6) in systemic 
circulation[6],which has been strongly linked to the neuroinflammatory cascade that accompanies brain bold barrier failure[8]. In this case,pro-inflammatory cytokines and monocyte derived macrophages enter, leading to the activation of glial cells, including microglia and astrocytes[9, 10]. This process is mainly affected by bone marrow-derived macrophages (BMDMs), which enable microglia/macrophages to play a dual role in the microenvironment of brain injury and repair[11, 12]. Upon microglial activation, the inflammatory cascade is triggered by the release of pro-inflammatory molecules and concomitant signalling pathways are activated, which causes synaptic damage, neuronal loss and progression. Reactive astrocytes exhibit neurotoxic effects with loss of neurotrophic functions[13]. The interaction between peripheral immunity and brain caused by systemic inflammation amplifies the inflammatory response in the central nervous system (CNS)[14], while the cascade of neuroinflammation induces synaptic dysfunction and neuronal apoptosis, finally damaging cognitive function[6, 15].

Advances on mechanisms in resolution of acute inflammation uncovered a new genus of pro-resolving lipid mediators, called specialized pro-resolving mediators (SPM)[16], which can increased by Netrin-1 (NTN-1) in vivo during acute self-limited inflammation[17]. NTN-1 is an axonal guidance molecule, involved in both physiological and pathological processes such as apoptosis, inflammation and neurogenesis in the nervous system as well as in the lung, heart, and kidneys. NTN-1 has been shown to play a positively regulatory role during inflammatory process recently[18]. It has been demonstrated that NTN-1 can limit inflammatory response through the involvement of inflammatory cascades[19]. In addition, NTN-1 was identified as a survival factor for endothelial cells and induced neovascularization and vessel remodeling. Overexpression of NTN-1 promoted angiogenesis and improved long-term neurological functions following ischemic stroke. Recent studies indicated that NTN-1 preserved BBB integrity in model of traumatic brain injury and experimental autoimmune encephalomyelitis[20, 21]. However, there are not any reports about the role of NTN-1 in the POD.

Based on these discoveries, we proposed the hypothesis that pretreatment with NTN-1 could improve the POD-like behavior of aged mice through its anti-inflammation effect on the inflammation induced by surgical trauma. To validate this hypothesis, we assessed the effects of NTN-1 on the postoperative behavior of aged mice, and inflammation events both in the periphery and CNS. In addition, we aimed to determine that NTN-1 prevents peripheral inflammatory factors from entering the brain by protecting the tightness of the blood-brain barrier, which plays an important role in preventing peripheral inflammation from metastasizing to CNS.

\section{Materials And Methods}

\section{Animals}

All procedures were approved by the Animal Ethics Committee of Zhongnan Hospital of Wuhan University, Hubei, China, and all experiments were performed in accordance with the National Institutes of Health Guidelines for the Care and Use of Laboratory Animals. Efforts were made to minimize the number of animals used. C57BL/6J mice (18-month-old, female, weighing 30-40 g) were purchased from 
Changsha Tianqin Biotechnology Co. Ltd. Changsha, China. All animals were group-housed five per cage with free access to food and water. The temperature, humidity, and day-night cycle were maintained according to the standards established by the experimental animal laboratory at Zhongnan Hospital of Wuhan University. The mice were allowed one week to acclimatize the laboratory environment before the experiment.

\section{Experimental protocol}

Mice were randomly divided into 4 groups: control group, surgery group, surgery + netrin-1 group, and netrin-1 group. Netrin-1 (R\&D Systems, 6419-N1-025) was given at $45 \mu \mathrm{g} / \mathrm{kg}$ in phosphate-buffered saline (PBS), and administered through tail vain with a total volume of $200 \mu \mathrm{L}$ at 1 hour after surgery, while the equal volume of PBS was given in control group and surgery group. The dose of netrin-1 was based on the researches using other models of acute inflammation with slight modification[21].

\section{POD mouse model}

A simple laparotomy was performed under isoflurane anesthesia using the methods described in our previous studies[22]. Specifically, anesthesia was induced and maintained with $1.4 \%$ isoflurane in $100 \%$ oxygen in a transparent acrylic chamber. Fifteen minutes after the induction, the mouse was moved out of the chamber, and isoflurane anesthesia was maintained via a cone device. One 16-gauge needle was inserted into the cone near the nose of the mouse to monitor the concentration of isoflurane. $\mathrm{A}$ longitudinal midline incision was made from the xiphoid to the 0.5 centimeter proximal pubic symphysis on the skin, abdominal muscles and peritoneum. Then, the incision was sutured layer by layer with $5-0$ Vicryl thread. At the end of the procedure, EMLA cream (2.5\% lidocaine and $2.5 \%$ prilocaine) was applied to the incision wound, and then every eight hours for two days to treat the pain associated with the incision. The procedure for each mouse lasted about ten minutes, and the mouse was put back into the anesthesia chamber for up to 2 hours to receive the rest of the anesthesia consisting of $1.4 \%$ isoflurane in $100 \%$ oxygen. A heat pad was used to keep the mouse body temperature between $36^{\circ} \mathrm{C}$ and $37^{\circ} \mathrm{C}$ during the surgery. After recovering from the anesthesia, each mouse was returned to a home house with available food and water.

\section{Behavioral tests}

The behavioral changes were detected using battery of behavioral tests including buried food test, open field test, and finally $Y$ maze test-at 24 hours before (baseline) the Surgery/Anesthesia and at 6, 9, 24 hours postoperatively as described in our previous studies[23]. In all tests, apparatus was cleaned with $75 \%$ alcohol after each mouse to remove odors.

\section{1 Buried food test}

The buried food test was performed as described in previous studies[22, 24] with modifications. Specifically, two days before buried food test, each mouse was received 2 pieces of sweetened cereal. On the test days, we had the each mouse acclimatize for one hour by placing the home cage with mice in the testing room. The test cage was prepared with clean paddling of 3 centimeters high in which we buried 1 piece of sweetened cereal below the padding. Its location was freely chosen and it was not visible. We 
placed the mouse in the center of the cage and measured the latency of eating the food. The latency was defined as the time from when the mouse was placed in the case to the mouse uncovered the food and grasped it with forepaws and/or teeth. When mice found the food pellet within 5 minutes, they were allowed to eat up the food and then returned their home cage. If they couldn't find the pellet within 5 minutes, they would be taken back when up to 5 minutes and the latency was recorded as 300 seconds.

\subsection{Open field test}

The open field test was performed as described in previous studies[23, 25] with modifications. Specifically, the mouse was placed in the center of an open field chamber ( $40 \times 40 \times 40$ centimeters) under dim light and was allowed to move freely for 5 minutes. The activities were automatically recorded by a video camera connected to the Any-Maze animal tracking system software (Xinruan Information Technology Co. Ltd., Shanghai, China), and movement parameters were calculated by the software. The total distance moved (meters), the time (seconds) spent in the center of the open field, the freezing time (seconds) and the latency (the time in seconds for the mice to reach to the location at the first attempt) to the center of the open field were recorded and analyzed.

\subsection{Y maze test}

The $Y$ maze test was performed as described in previous studies with modifications[26, 27]. Specifically, the $Y$ maze was placed in a quiet and illuminated room, and consisted of three arms $(8 \times 30 \times 15$ centimeters) with an angle of 120 degrees between each arm. The three arms included the start arm, in which the mouse started to explore (always open), novel arm, which was blocked at the first trial, but opened at the second trial, and the other arm (always open). The start arm and other arm were designed randomly to avoid the spatial memory error. The $Y$ maze test consisted of two trials separated by an intertrial interval (ITI). The first trial (training) was 10 minutes in duration and allowed the mouse to explore start arm and other arm. After 2 hours (for the studies of 6 and 24 hours after the surgery) or 4 hours (for the study of 9 hours after surgery) ITI, the second trial was conducted. For the second trial, the mouse was placed back in the maze in the same start arm with free access to all arms for 5 minutes. A video camera, which was linked to the Any-Maze animal tracking system software, was installed 60 centimeters above the chamber to monitor and analyze the number of entries and the time spent in each arm. The time spent in and entries into the novel arms indicated the spatial recognition memory (learned behavior).

\section{Enzyme-linked Immune-sorbent Assay (ELISA)}

The mouse IL-6 ELISA kit (ELK Biotechnology, ELK1157), mouse IL-10 ELISA kit (ELK Biotechnology, ELK1143), mouse HMGB-1 ELISA kit (ELK Biotechnology, ELK1440), mouse netrin-1 (NTN-1) ELISA kit (CUSABIO, CSB-EL016127MO) were used to evaluate peripheral or central inflammation and the levels of netrin-1 in brain tissue at 6 hours postoperative.

\section{Western blot analysis}

Hippocampus and prefrontal cortex of the mice were harvested at 9 hours after surgery. Anti-ZO-1 (1:500, Abcam, ab96587), anti-occludin (1:2000, Abcam, ab167161), and anti-claudin-5 (1:500, Biorbyt, orb214680) were used to detect the expression of tight junction-associated proteins in hippocampus and 
prefrontal cortex. Anti- $\beta$-actin (1:10,000, TDY Biotech, ab37168) was used to normalized and control for loading differences in the protein levels. The bands were measured using image analysis software (AlphaEaseFC software), and changes in protein levels were presented as folds of those in the control group.

\section{BBB Permeability Assay}

Dextran was used to Dextran was used to measure BBB permeability as described in previous studies with modifications[28, 29]. Specifically, 6 hours after surgery, each mouse was injected intravenously with $100 \mu \mathrm{l}$ 10-kDa dextran Texas Red lysine fixable (4 mg/ml, Invitrogen, D1863). Fifteen minutes after injection, each mouse was anesthetized with $1.4 \%$ isoflurane and decapitated. The brain tissue was harvested and fixed by $4 \%$ paraformaldehyde overnight at $4{ }^{\circ} \mathrm{C}$, then cryopreserved in $30 \%$ sucrose and frozen in TissueTek OCT (Sakura). Frozen sections of $20 \mu \mathrm{m}$ were collected and post-fixed in 4\% PFA at room temperature $\left(20-25^{\circ} \mathrm{C}\right)$ for $15 \mathrm{~min}$, washed in PBS and were blocked with $10 \%$ goat serum (Boster Biologic Technology, China) for 2 hours, permeabilized with $0.5 \%$ Triton X-100, then incubated with isolectin B4 (20 $\mathrm{\mu g} / \mathrm{ml}, 121411$, Molecular Probes, San Francisco, CA, USA) for immunostaining to visualize blood vessels. Zeiss LSM 510 META microscope was used to detect the fluorescence images of the injected tracer and isolectin under $40 \times$ objective lens. For each mouse, 20 images of 10 different slices of hippocampus and prefrontal cortex were randomly selected, and the level of dextran founded outside the vessels were analyzed using ImageJ $(\mathrm{NIH})$.

Spectrophotometric quantification of 10-kDa dextran Texas Red from extracts of hippocampus and prefrontal cortex was carried out at 24 hours after surgery. Specifically, each mouse was injected intravenously with $100 \mu$ l 10-kDa dextran Texas Red lysine fixable $(4 \mathrm{mg} / \mathrm{ml}$, Invitrogen, D1863) 24 hours postoperatively. Fifteen minutes after injection, each mouse was deeply anesthetized and perfused with PBS transcardially ( $150 \mathrm{~mL}$ for $5 \mathrm{~min}$ ). Then the mice were decapitated, and the hippocampus and prefrontal cortex were harvested. Then we used 1\% Triton X-100 in PBS to homogenize the brain tissue (100 $\mu \mathrm{L} / 100 \mathrm{mg}$ brain tissue). Tissue lysates were centrifuged at 16000 r.p.m. for 20 minutes and the fluorescence of the supernatant was measured on a fluorometer POLAR star Omega (BMG Labtech) (ex/em 595/615 nm).

\section{Immunofluorescence}

24 hours after surgery, each mouse was anesthetized with $1.4 \%$ isoflurane and perfused transcardially with ice-cold 0.1 M PBS followed by $4 \%$ PFA in $0.1 \mathrm{M} \mathrm{PBS}$ at pH 7.4. Brains were harvested and fixed in $4 \%$ PFA in $0.1 \mathrm{M}$ PBS at $4{ }^{\circ} \mathrm{C}$, then cryoprotected in $30 \%$ sucrose for 72 hours, and frozen in TissueTek OCT (Sakura), were cut sequentially to $20 \mu \mathrm{m}$. Washed in PBS and permeabilized in $0.5 \%$ Triton X-100, the section was blocked with $10 \%$ goat serum for 2 hours at room temperature in order to block non-specific bindings, washed in PBS, then the following primary antibody rabbit anti-Iba-1 (1:200, Abcam, ab178847) at $4{ }^{\circ} \mathrm{C}$ overnight. After washing, the sections were incubated with secondary antibody (goat anti-rabbit) conjugating with Alexa Fluor dyes 488 from Invitrogen (1:500) at room temperature for 2 hours at dark. Immunolabelled sections were coverslipped with 40,6-diamidino-2-phenylindole (DAPI; Invitrogen) and analyzed by microscope (Olympus, Tokyo, Japan) equipped with an imaging system. Five high 
magnifications were chosen in three non-overlapping fields randomly acquired in hippocampus and prefrontal cortex subregions using a counting frame size of $0.4 \mathrm{~mm}^{2}$. Images were processed and the area of the microglia quantified using ImageJ software $(\mathrm{NIH})$. The area of the selected cells was converted into a immunoreactivity was calculated as percentage area density defined as the number of pixels (positively stained area) divided by the total number of pixels (sum of positively and negatively stained area) in the imaged field.

\section{Statistical Analysis}

The statistical analysis were performed with SPSS 23.0 (IBM, New York, USA) or GraphPad Prism 6 (GraphPad, New York, USA). The normality of the data was analyzed using the Shapiro-Wilk test, and the data was found to be normally distributed. The quantitative data are expressed as the mean \pm standard error of the mean (SEM), with the error bars indicating the SEM. Different groups were compared using the one-way analysis of variance, followed by Bonferroni post hoc test. A value of $p<0.05$ was considered statistically significant.

\section{Results}

\section{Administration of exogenous NTN-1 improves POD-like behavior induced by surgery/anesthesia in aged mice}

To assess whether surgery/anesthesia affects general and cognitive behavior of aged mice, we performed a battery of behavioral tests with food buried test, open field test and $\mathrm{Y}$ maze test, at 24 hours before surgery and 6, 9, 24 hours after surgery in the present study as we previously reported[22, 23]. Composite $Z$ scores for each of the 40 mice in the four groups were calculated at 6,9 and 24 hours after surgery $(P<0.05$, Fig. $1 \mathrm{~A}-\mathrm{C})$.

At first we executed the buried food test to explore whether surgery/anesthesia affected the mice's ability to associate odorant with food reward[30]. The latency to eat food was markedly increased in the Surgery group compared to the Control group at 9 hours after surgery $(P<0.01$, Fig. 1D), while administration with NTN-1 improved the impaired ability of finding and eating food induced by surgery/anesthesia $(P<0.05$, Fig. 1D). No significant changes were observed between the NTN-1 group and Control group.

Surgery/anesthesia-induced impairment in mice's ability to search for and eat food suggests that the surgery/anesthesia might cause the mice to develop the changes in behaviors (inattention, disorganized thinking and altered level of consciousness) associated with delirium.

Then we executed the open field test to examine the locomotor ability and exploratory behavior of mice. There were no significant differences in total distance traveled by mice between four groups at 9 hours after surgery, indicating that surgery/anesthesia did not affect the motor function of aged mice (Fig. 1E). Surgery/anesthesia significantly decreased the time spent in the center at 9 hours after surgery $(P<0.05$, Fig. 1F), and preemptive administration of NTN-1 ameliorated this phenomenon at 9 hours after surgery $(P<0.05$, Fig. 1F). Besides, surgery/anesthesia significantly decreased the freezing time at 9 hours after 
surgery $(P<0.05$, Fig. $1 \mathrm{G})$, while preoperative treatment with NTN-1 increased the freezing time at 9 a hours after surgery $(P<0.05$, Fig. 1G). It's worth noting that NTN-1 administration did not change these parameters compared to the control condition (Fig. 1E-G). These findings suggest that the surgery/anesthesia altered the natural behavior of the mice such as anxiety (time spent in the center) and natural reaction (freezing time).

At last we conducted $Y$ maze for assessing the spatial memory in aged mice as previously validated[31]. Surgery/anesthesia significantly reduced the number of entries in the novel arm at 9 hours after surgery $(P<0.05$, Fig. $1 \mathrm{H})$ and the duration in the novel arm at 9 hours after surgery $(P<0.05$, Fig. $1 \mathrm{I})$, as compared to the control condition. Pretreatment with NTN-1 increased the number of entries in the novel arm and duration in the novel arm at 9 hours after surgery $(P<0.05$, Fig. $1 \mathrm{H}-\mathrm{I})$. However, NTN-1 administration alone did not affect the performance of aged mice in the $Y$ maze test at 9 hours after surgery (Fig. $1 \mathrm{H}-\mathrm{I})$.

Taken together, no significant changes were observed between the NTN-1 group and Control group but prophylaxis with NTN-1 attenuated the impairment of POD-behavior by surgery/anesthesia of aged mice in a fluctuating way.

\section{NTN-1 regulates the expression of inflammatory cytokines after surgery}

To evaluate the effects of NTN-1 on the systemic inflammation, we firstly measured the changes of IL-6, IL-10 and HMGB-1 in blood plasma at 6 hours after surgery[32]. Surgery/anesthesia significantly increased the level of IL- 6 and HMGB-1(P凶0.05, Fig. 2A,2C)but did not change the expression of IL-10 after surgery ( $P \otimes 0.05$, Fig. 2B). Though a single dose of NTN-1 did not completely reverse the increase of proinflammatory cytokines to the control condition, it markedly reduced the levels of IL- 6 and HMGB-1 after surgery $(P \otimes 0.05$, Fig. 2A,2C). Besides, pretreatment of NTN-1 increased the expression of IL-10, a crucial cytokine during the resolution phase of inflammation after surgery $(P \otimes 0.05$, Fig. 2B). Secondly, we measured these cytokines above in the hippocampus and prefrontal cortex which are two key brain regions related to memory network $[33,34]$ to evaluate the effects of NTN-1 on neuroinflammation at 6 hours after surgery. Surgery/anesthesia induced a marked increase in the expression of IL- 6 after surgery both in the hippocampus and prefrontal cortex compared to the control condition ( $P \otimes 0.05$, Fig. 3A, 3D).

Pretreatment with NTN-1 significantly decreased the expression of IL-6 compared to the Surgery group in these brain regions $(P \otimes 0.05$, Fig. 3A,3D). Besides, pretreatment with NTN-1 increased the expression of IL10 not only in the hippocampus after surgery $(P \otimes 0.05$, Fig. 3B), but also in the prefrontal cortex after surgery $(P \otimes 0.05$, Fig. 3E).

\section{Surgery/anesthesia decreases the endogenous NTN-1 in the hippocampus and the prefrontal cortex in aged mice}

To investigate whether the endogenous NTN-1 was involved in anti-inflammatory and neuroprotective effects, we measured the changes of the endogenous NTN-1 in the hippocampus and the prefrontal cortex at 6 hours after surgery. Our result suggested that surgery/anesthesia significantly decreased the level of NTN-1 in the hippocampus and the prefrontal cortex after surgery (P®0.05, Fig. 3C,3F). 


\section{NTN-1 prevents neuroinflammation in the hippocampus and prefrontal cortex}

We measured the changes of immunoreactivity of Iba-1 in the hippocampus and prefrontal cortex to assess the reactive states of microglia, which represent the major pathological manifestation of neuroinflammation[35, 36]. NTN-1 attenuated microglial activation as measured by changes in the expression of Iba-1.Surgery induced the amoeba-like morphology of microglia and increased Iba-1 immunoreactive area in the hippocampus and prefrontal cortex compared with the control condition ( $P \otimes 0.05$, Fig. 4A-D), while preemptive administration of NTN-1 significantly restored the ramified shape of microglia and reduced cellular area $(P \otimes 0.05$, Fig. 4A-D). No significant changes in lba-1 were observed in the NTN-1 group.

\section{NTN-1 prophylaxis alleviates the leakage of BBB induced by surgery/anesthesia}

The breakdown of blood-brain barrier (BBB) has been reported to be associated with delirium and perioperative neurocognitive disorders[37,38], so we employed a well-established dye injection assay to investigate the integrity of $\mathrm{BBB}[32,39]$ under the treatment of surgery/anesthesia with or without administration of NTN-1.

The immunofluorescence images revealed that $10-\mathrm{kDa}$ dextran was primarily confined to vessels in the four groups. By contrast, the signal of dextran was detected in the brain parenchyma around vessels of mice in the Surgery group (Fig. 5A). To quantitate the extravascular dextran, spectrophotometric quantification of 10-kDa dextran-Texas Red from brain tissue extracts was performed. In the hippocampus, we found that surgery/anesthesia increased the level of extravascular 10-kDa dextran as compared to the control condition, while NTN-1 prophylaxis decreased the leakage of dextran induced by surgery/anesthesia ( $P<0.05$, Fig. 5B).

We next examined the effects of NTN-1 on the expression of occludin, ZO- 1 and claudin- 5 after surgery (Fig. 6D-F, Fig. 7D-F), which are the tight junction (TJ) associated proteins to maintain the integrity of $\mathrm{BBB}[40,41]$. By quantitative western blot we found that there was a marked decrease in the expression of occludin, ZO-1 and claudin- 5 both in the hippocampus and prefrontal cortex at 9 hours after surgery, while pretreatment with NTN-1 significantly attenuated the reduction of these proteins $(P<0.05$, Fig. 6A-C, Fig. 7A-C,). Preemptive administration of NTN-1 alone did not have any effects on BBB.

\section{Discussion}

In the present study, we demonstrate that the exogenous NTN-1, an axonal guidance molecule, improve the postoperative of POD-like behavior in aged mice by its anti-inflammatory and BBB-protecting effect. Our results indicate that pretreatment with NTN-1 given through the caudal vein alleviates systemic inflammatory response and protects BBB integrity after surgery/anesthesia. In addition, the exogenous NTN-1limits neuroinflammation both in the hippocampus and prefrontal cortex, according to the expression of inflammatory cytokines and reactive states of microglia in these brain regions. As far as we know,this is the first report about the neuroprotective effect of NTN-1 in mice model of POD. 
A large amount of evidence indicated that neuroinflammation plays an important role in POD. Peripheral aseptic inflammation activates innate immune system, which starts the inflammatory process and eventually lead to POD.[7, 15, 42]. In the aseptic surgery setting, cell trauma releases damage associated molecular patterns (DAMPs) that bind to Toll-like receptors (TLRs) via high mobility group box-1 (HMGB1) to activate BMDMs, which then upregulating the expression of pro-inflammatory cytokines such as TNF-a, IL-1 and IL-6[43, 44]. These cytokines can cause further activation of DAMPs in positive feedback $[45,46]$ and be released into the circulation and disrupt the integrity of the blood brain barrier (BBB) $[42,47]$. Our results show that NTN-1 attenuates the systemic release of proinflammatory factor IL-6 and increases of anti-inflammation cytokine IL-10 after surgery, which are the vital cytokines after trauma. At the same time, NTN-1 reduced the release of HMGB-1, which is passively released from cells damaged by aseptic trauma and targets circulating BM-DMs. These findings are consistent with the potent anti-inflammatory activity of NTN-1 in many other disease models that associated with inflammation such as renal ischemia reperfusion injury[48], acute peritonitis[49], acute pancreatitis[50]. The migration and aggregation of white blood cells to the inflammatory site is the central link of the whole inflammatory response. Early studies found that NTN-1 interacts with the UNC-5B receptor expressed on the surface of white blood cells and inhibits the migration of white blood cells[19]. In Alzheimer's disease (AD) rats[51], it has been demonstrated that NTN-1 concentrations in the serum were positively correlated with the systemic expression of IL-10, one of the most important mediators in the anti-inflammatory activity[52]. What is more, in acute peritonitis and acute colitis models, NTN-1 inhibits the migration of inflammatory cells and induces the M2 polarization phenotype of macrophages[53,54]. This further indicates that the changes of peripheral inflammatory factors may be related to powerful anti-inflammatory effect of NTN-1.

An intact functioning blood-brain barrier (BBB) is fundamental to proper homoeostatic maintenance and perfusion of the CNS. Inflammatory damage to the unique microvascular endothelial cell monolayer that constitutes the luminal BBB surface, leading to elevated capillary permeability, has been linked to various neurological disorders ranging from ischaemic stroke and traumatic brain injury, to neurodegenerative disease and CNS infections[55]. Moreover, the neuroinflammatory cascade that typically accompanies BBB failure in these circumstances has been strongly linked to elevated levels of proinflammatory cytokines such as tumor necrosis factor- $a$ (TNF-a) and interleukin-6 (IL-6)[8]. In models of subarachnoid hemorrhage[21], multiple sclerosis [56] and stroke[57], NTN-1 has been shown to have a protective effect on BBB and improve neurocognitive function, which was also noted in our model. There is compelling evidence that exogenous NTN-1 significantly diminished the diffusion of dextran across mouse brainderived endothelial cells in vitro. The barrier tightening induced by NTN-1, at least in part, is the consequence of netrin-induced tight junction molecule upregulation. It has been reported that levels of both transmembrane and intracellular components of the junctional complex increased in response to NTN-1. In addition, treatment of human brain-derived endothelial cells with NTN-1 enriched junctional proteins in lipid raft membrane microdomains, where proteins effectively interact to form functional clusters that support barrier integrity[20]. Thus, NTN-1 reduces the incidence of POD by reducing the entry of peripheral inflammatory cytokines through impaired flow barriers. 
In addition to mitigate peripheral inflammatory response, NTN-1 reduces the activation of glia cells and the expression of inflammatory cytokines in the hippocampus and prefrontal cortex. Microglia are crucially important during development involved in the phagocytosis of neural precursor cells[58]. Under non-injurious conditions, microglia subserve important functions involved in surveillance of brain parenchyma in order to maintain homeostasis[59]. Following release of pro-inflammatory cytokines by the innate immune response, microglia are activated by one or more pathways. Activated microglia rapidly switch to a proinflammatory phenotype with stout morphology, and enhance the production of proinflammatory molecules [42]. These pro-inflammatory cytokines and the debris released by activated microglia can convert astrocytes into a neurotoxic A1 reactive subtype[60,61], which cause astrocytes to lose their normal synaptic maintenance and phagocytosis along with induce rapid death of neurons and oligodendrocytes[60, 61]. In our model of POD, NTN-1 reverts the morphological changes of microglia both in the hippocampus and prefrontal cortex to their original forms, representing the transformation of the inflammatory phenotype to the resting state, thereby changing the pro-inflammatory environment by regulating the secretion of inflammatory cytokines. Herein, it is reasonable that pre-treatment with NTN-1 facilitates the improve of POD-like behavior in aged mice because hippocampus and prefrontal cortex which are in charge of shaping emotion, learning and organizing memory $[62,63]$.

In addition, the regulation of lipid mediators by neuronal circuits might be an important part in the control of inflammation to sterile injury. The vagus nerve regulates the expression of the axonal guidance molecule NTN-1 can increases SPM production in vivo during acute-self limited inflammation, were this protein upregulates exudate RvD5 and PD1 concentrations[17, 64]. Our previous research has verified the anti - inflammatory and proresolving activities of PD1 in the inflammatory milieu both in vivo and in vitro and identified the role of PD1 in regulating postoperative inflammation and ensuing POD-like behavior of mice. In our study, compared with the control group, the concentration of the endogenous NTN-1 in hippocampus and prefrontal cortex at 6 hours postoperatively significantly reduced. This is most likely the result of the endogenous NTN-1 being consumed after participating in pro-resolution of inflammation by regulating SPM. So, the neuroprotective effect of Netrin-1 may be related to this mechanism. What is more, NTN-1 is involved in regulating inflammatory signaling pathways and inhibiting the production of pro-inflammatory cytokines. In previous studies, it has been observed that NTN-1 can promote the production of CAMP in immune cells and activate the CAMP/ protein kinase A (PKA) signaling pathway to inhibit the production of pro-inflammatory cytokines $[65,66]$. Ranganathan found that inhibits the ischemia-reperfusion(I/R) induced acute kidney injury (AKI) NTN-1 model of renal tubular epithelial cells, polymorphonuclear neutrophils(PMN) and mononuclear cells in an enzyme called cyclooxygenase 2 (cox2) expression. NTN-1 May inhibit the NF-KB activation lowered cox-2 expression, thus reduce the inflammatory response[67]. Does NTN-1 also inhibit the production of pro-inflammatory mediators by other means? It is thus essential to explore the underlying mechanism of NTN-1 on inflammation in further investigation.

There are several limitations to our research. First of all, there are a number of signaling pathways that have been shown to be involved in anti-inflammatory and vascular endothelial cell protection. An in-depth study of the mechanism of NTN-1 that we need to search will open up a new way for the prevention and 
treatment of inflammation-related lesions. Secondly, we have only demonstrated that exogenous prophylactic NTN-1 can improve POD by providing positive anti-inflammatory responses and protective BBB functions after surgery in elderly mice. However, how endogenous NTN-1 changes during this process has not been studied, NTN-1 small interfering RNA (siRNA) can be used in later study.

\section{Conclusions}

In conclusion, the present study identifies the administration of exogenous NTN-1 could regulate postoperative inflammation and protect the integrity of BBB to improve POD of aged mice. These findings indicate the potential of NTN-1 to be a novel therapy for POD.

\section{Abbreviations}

POD: postoperative delirium

IL-6: interleukin-6

IL-10: interleukin-10

HMGB-1: high mobility group box chromosomal protein-1

BBB: blood-brain barrier

TNF-a: necrosis factor- $a$

BMDMs: bone marrow-derived macrophages

CNS: central nervous system

SPM: specialized pro-resolving mediators

NTN-1: Netrin-1

PBS: phosphate-buffered saline

TJ: tight junction

DAMPs: damage associated molecular patterns

TLRs: Toll-like receptors

AD: Alzheimer's disease

PKA: protein kinase $A$

I/R: ischemia-reperfusion 
AKI: acute kidney injury

PMN: polymorphonuclear neutrophils

siRNA: small interfering RNA

\section{Declarations}

\section{Ethics approval and consent to participate}

All animal experiments were approved by Animal Ethics Committee of Zhongnan Hospital of Wuhan University, Hubei, China.

\section{Consent for publication}

Not applicable.

\section{Availability of data and materials}

All data generated during this study are included in this published article. Further details regarding the presented datasets are available from the corresponding author upon request.

\section{Competing interests}

The authors declare no competing interests.

\section{Funding}

This research was supported by the grants from National Natural Science Foundation of China (81371195, 81870851 and 82071208), and the Outstanding Talented Young Doctor Program of Hubei Province (HB20200407).

\section{Authors' contributions}

$\mathrm{KL}$ and JW designed and performed the experiment, collected and analyzed the data, and prepared the manuscript. $M G$ and $X L$ were involved in preparing the animal models and participated in interpreting the results. $\mathrm{LC}$ contributed to behavioral testing. $\mathrm{YZ}$ was involved in biochemical analysis. $\mathrm{KL}$ and JW participated in the statistical analysis. MP contributed to the study concept and design, secured funding for the project, and prepared and critically revised the manuscript. All authors reviewed the manuscript.

\section{Acknowledgements}

Not applicable.

\section{References}


1. Auerbach RP, Mortier P, Bruffaerts R, Alonso J, Benjet C, Cuijpers P, Demyttenaere K, Ebert DD, Green JG, Hasking P, et al: WHO World Mental Health Surveys International College Student Project: Prevalence and distribution of mental disorders. J Abnorm Psychol 2018, 127:623-638.

2. Aldecoa C, Bettelli G, Bilotta F, Sanders RD, Audisio R, Borozdina A, Cherubini A, Jones C, Kehlet H, MacLullich A, et al: European Society of Anaesthesiology evidence-based and consensus-based guideline on postoperative delirium. Eur J Anaesthesiol 2017, 34:192-214.

3. Androsova G, Krause R, Winterer G, Schneider R: Biomarkers of postoperative delirium and cognitive dysfunction. Front Aging Neurosci 2015, 7:112.

4. Whitlock EL, Vannucci A, Avidan MS: Postoperative delirium. Minerva Anestesio/ 2011, 77:448-456.

5. Marcantonio ER: In the clinic. Delirium. Ann Intern Med 2011, 154:ITC6-1, ITC6-2, ITC6-3, ITC6-4, ITC65, ITC6-6, ITC6-7, ITC6-8, ITC6-9, ITC6-10, ITC16-11, ITC16-12, ITC16-13, ITC16-14, ITC16-15; quiz ITC16-16.

6. Vacas S, Degos V, Feng X, Maze M: The neuroinflammatory response of postoperative cognitive decline. Br Med Bull 2013, 106:161-178.

7. Maldonado JR: Neuropathogenesis of delirium: review of current etiologic theories and common pathways. Am J Geriatr Psychiatry 2013, 21:1190-1222.

8. Rochfort KD, Cummins PM: The blood-brain barrier endothelium: a target for pro-inflammatory cytokines. Biochem Soc Trans 2015, 43:702-706.

9. Hu J, Feng X, Valdearcos M, Lutrin D, Uchida Y, Koliwad SK, Maze M: Interleukin-6 is both necessary and sufficient to produce perioperative neurocognitive disorder in mice. $\mathrm{Br} J$ Anaesth 2018, 120:537545.

10. Terrando N, Eriksson LI, Ryu JK, Yang T, Monaco C, Feldmann M, Jonsson Fagerlund M, Charo IF, Akassoglou K, Maze M: Resolving postoperative neuroinflammation and cognitive decline. Ann Neurol 2011, 70:986-995.

11. Xiong XY, Liu L, Yang QW: Functions and mechanisms of microglia/macrophages in neuroinflammation and neurogenesis after stroke. Prog Neurobio/ 2016, 142:23-44.

12. Prinz M, Priller J: Microglia and brain macrophages in the molecular age: from origin to neuropsychiatric disease. Nat Rev Neurosci 2014, 15:300-312.

13. Kaur D, Sharma V, Deshmukh R: Activation of microglia and astrocytes: a roadway to neuroinflammation and Alzheimer's disease. Inflammopharmacology 2019, 27:663-677.

14. Matsuda M, Huh Y, Ji RR: Roles of inflammation, neurogenic inflammation, and neuroinflammation in pain. $J$ Anesth 2019, 33:131-139.

15. Girard S, Brough D, Lopez-Castejon G, Giles J, Rothwell NJ, Allan SM: Microglia and macrophages differentially modulate cell death after brain injury caused by oxygen-glucose deprivation in organotypic brain slices. Glia 2013, 61:813-824.

16. Serhan CN: Pro-resolving lipid mediators are leads for resolution physiology. Nature 2014, 510:92101. 
17. Dalli J, Serhan $\mathrm{CN}$ : Immunoresolvents signaling molecules at intersection between the brain and immune system. Curr Opin Immunol 2018, 50:48-54.

18. Tang Z, Xu C, Zhang G, Chen H: [Protect role of Netrin-1 in inflammatory response]. Zhonghua Wei Zhong Bing Ji Jiu Yi Xue 2016, 28:756-760.

19. Ly NP, Komatsuzaki K, Fraser IP, Tseng AA, Prodhan P, Moore KJ, Kinane TB: Netrin-1 inhibits leukocyte migration in vitro and in vivo. Proc Natl Acad Sci U S A 2005, 102:14729-14734.

20. Podjaski C, Alvarez JI, Bourbonniere L, Larouche S, Terouz S, Bin JM, Lecuyer MA, Saint-Laurent O, Larochelle C, Darlington PJ, et al: Netrin 1 regulates blood-brain barrier function and neuroinflammation. Brain 2015, 138:1598-1612.

21. Xie Z, Enkhjargal B, Reis C, Huang L, Wan W, Tang J, Cheng Y, Zhang JH: Netrin-1 Preserves BloodBrain Barrier Integrity Through Deleted in Colorectal Cancer/Focal Adhesion Kinase/RhoA Signaling Pathway Following Subarachnoid Hemorrhage in Rats. J Am Heart Assoc 2017, 6.

22. Lu Y, Chen L, Ye J, Chen C, Zhou Y, Li K, Zhang Z, Peng M: Surgery/Anesthesia disturbs mitochondrial fission/fusion dynamics in the brain of aged mice with postoperative delirium. Aging (Albany NY) 2020, 12:844-865.

23. Peng $M$, Zhang $C$, Dong $Y$, Zhang $Y$, Nakazawa $H$, Kaneki $M$, Zheng $H$, Shen $Y$, Marcantonio ER, Xie Z: Battery of behavioral tests in mice to study postoperative delirium. Sci Rep 2016, 6:29874.

24. Zhang J, Gao J, Guo G, Li S, Zhan G, Xie Z, Yang C, Luo A: Anesthesia and surgery induce deliriumlike behavior in susceptible mice: the role of oxidative stress. Am J Transl Res 2018, 10:2435-2444.

25. Meng B, Li X, Lu B, Liu R, Yuan H, Zhai X, Qin J, Chen Z, Zheng J, Chen J: The Investigation of Hippocampus-Dependent Cognitive Decline Induced by Anesthesia/Surgery in Mice Through Integrated Behavioral Z-Scoring. Front Behav Neurosci 2019, 13:282.

26. Wolf A, Bauer B, Abner EL, Ashkenazy-Frolinger T, Hartz AM: A Comprehensive Behavioral Test Battery to Assess Learning and Memory in 129S6/Tg2576 Mice. PLoS One 2016, 11:e0147733.

27. Chen Y, Liu X, Jia X, Zong W, Ma Y, Xu F, Wang J: Anxiety- and depressive-like behaviors in olfactory deficient Cnga2 knockout mice. Behav Brain Res 2014, 275:219-224.

28. Kutuzov N, Flyvbjerg $\mathrm{H}$, Lauritzen M: Contributions of the glycocalyx, endothelium, and extravascular compartment to the blood-brain barrier. Proc Natl Acad Sci U S A 2018, 115:E9429-E9438.

29. Bernstein DL, Zuluaga-Ramirez V, Gajghate S, Reichenbach NL, Polyak B, Persidsky Y, Rom S: miR-98 reduces endothelial dysfunction by protecting blood-brain barrier (BBB) and improves neurological outcomes in mouse ischemia/reperfusion stroke model. J Cereb Blood Flow Metab 2020, 40:19531965.

30. Yang M, Crawley JN: Simple behavioral assessment of mouse olfaction. Curr Protoc Neurosci 2009, Chapter 8:Unit 824.

31. Wheelan N, Webster SP, Kenyon CJ, Caughey S, Walker BR, Holmes MC, Seckl JR, Yau JL: Short-term inhibition of 11beta-hydroxysteroid dehydrogenase type 1 reversibly improves spatial memory but persistently impairs contextual fear memory in aged mice. Neuropharmacology 2015, 91:71-76. 
32. Yang S, Gu C, Mandeville ET, Dong Y, Esposito E, Zhang Y, Yang G, Shen Y, Fu X, Lo EH, Xie Z: Anesthesia and Surgery Impair Blood-Brain Barrier and Cognitive Function in Mice. Front Immunol 2017, 8:902.

33. Place R, Farovik A, Brockmann M, Eichenbaum H: Bidirectional prefrontal-hippocampal interactions support context-guided memory. Nat Neurosci 2016, 19:992-994.

34. Preston AR, Eichenbaum H: Interplay of hippocampus and prefrontal cortex in memory. Curr Biol 2013, 23:R764-773.

35. Joshi AU, Minhas PS, Liddelow SA, Haileselassie B, Andreasson KI, Dorn GW, 2nd, Mochly-Rosen D: Fragmented mitochondria released from microglia trigger A1 astrocytic response and propagate inflammatory neurodegeneration. Nat Neurosci 2019, 22:1635-1648.

36. Norden DM, Trojanowski PJ, Villanueva E, Navarro E, Godbout JP: Sequential activation of microglia and astrocyte cytokine expression precedes increased lba-1 or GFAP immunoreactivity following systemic immune challenge. Glia 2016, 64:300-316.

37. Subramaniyan S, Terrando N: Neuroinflammation and Perioperative Neurocognitive Disorders. Anesth Analg 2019, 128:781-788.

38. Maldonado JR: Pathoetiological model of delirium: a comprehensive understanding of the neurobiology of delirium and an evidence-based approach to prevention and treatment. Crit Care Clin 2008, 24:789-856, ix.

39. Ben-Zvi A, Lacoste B, Kur E, Andreone BJ, Mayshar Y, Yan H, Gu C: Mfsd2a is critical for the formation and function of the blood-brain barrier. Nature 2014, 509:507-511.

40. Jiao H, Wang Z, Liu Y, Wang P, Xue Y: Specific role of tight junction proteins claudin-5, occludin, and Z0-1 of the blood-brain barrier in a focal cerebral ischemic insult. J Mol Neurosci 2011, 44:130-139.

41. Luissint AC, Artus C, Glacial F, Ganeshamoorthy K, Couraud PO: Tight junctions at the blood brain barrier: physiological architecture and disease-associated dysregulation. Fluids Barriers CNS 2012, 9:23.

42. Saxena S, Maze M: Impact on the brain of the inflammatory response to surgery. Presse Med 2018, 47:e73-e81.

43. Levy RM, Mollen KP, Prince JM, Kaczorowski DJ, Vallabhaneni R, Liu S, Tracey KJ, Lotze MT, Hackam DJ, Fink MP, et al: Systemic inflammation and remote organ injury following trauma require HMGB1. Am J Physiol Regul Integr Comp Physiol 2007, 293:R1538-1544.

44. Li RL, Zhang ZZ, Peng M, Wu Y, Zhang JJ, Wang CY, Wang YL: Postoperative impairment of cognitive function in old mice: a possible role for neuroinflammation mediated by HMGB1, S100B, and RAGE. J Surg Res 2013, 185:815-824.

45. Nishigaki A, Kawano T, Iwata H, Aoyama B, Yamanaka D, Tateiwa H, Shigematsu-Locatelli M, Eguchi S, Locatelli FM, Yokoyama M: Acute and long-term effects of haloperidol on surgery-induced neuroinflammation and cognitive deficits in aged rats. J Anesth 2019, 33:416-425.

46. Huang C, Irwin MG, Wong GTC, Chang RCC: Evidence of the impact of systemic inflammation on neuroinflammation from a non-bacterial endotoxin animal model. J Neuroinflammation 2018, 
15:147.

47. Li G, Liang X, Lotze MT: HMGB1: The Central Cytokine for All Lymphoid Cells. Front Immuno/ 2013, 4:68.

48. Wang W, Reeves WB, Ramesh G: Netrin-1 and kidney injury. I. Netrin-1 protects against ischemiareperfusion injury of the kidney. Am J Physiol Renal Physiol 2008, 294:F739-747.

49. Ariel A, Fredman G, Sun YP, Kantarci A, Van Dyke TE, Luster AD, Serhan CN: Apoptotic neutrophils and T cells sequester chemokines during immune response resolution through modulation of CCR5 expression. Nat Immunol 2006, 7:1209-1216.

50. Chen J, Cai QP, Shen PJ, Yan RL, Wang CM, Yang DJ, Fu HB, Chen XY: Netrin-1 protects against LArginine-induced acute pancreatitis in mice. PLoS One 2012, 7:e46201.

51. Sun L, Ju T, Wang T, Zhang L, Ding F, Zhang Y, An R, Sun Y, Li Y, Lu Y, et al: Decreased Netrin-1 and Correlated Th17/Tregs Balance Disorder in Abeta1-42 Induced Alzheimer's Disease Model Rats. Front Aging Neurosci 2019, 11:124.

52. Mosser DM, Zhang X: Interleukin-10: new perspectives on an old cytokine. Immunol Rev 2008, 226:205-218.

53. Mirakaj V, Gatidou D, Potzsch C, Konig K, Rosenberger P: Netrin-1 signaling dampens inflammatory peritonitis. J Immunol 2011, 186:549-555.

54. Aherne CM, Collins CB, Masterson JC, Tizzano M, Boyle TA, Westrich JA, Parnes JA, Furuta GT, Rivera-Nieves J, Eltzschig HK: Neuronal guidance molecule netrin-1 attenuates inflammatory cell trafficking during acute experimental colitis. Gut 2012, 61:695-705.

55. Hawkins BT, Davis TP: The blood-brain barrier/neurovascular unit in health and disease. Pharmacol Rev 2005, 57:173-185.

56. Voortman MM, Pekar T, Bachmayer D, Archelos JJ, Stojakovic T, Scharnagl H, Ropele S, Pichler A, Enzinger $C$, Fuchs $S$, et al: Serum netrin-1 in relation to gadolinium-enhanced magnetic resonance imaging in early multiple sclerosis. Mult Scler J Exp Transl Clin 2017, 3:2055217317727294.

57. Yu J, Li C, Ding Q, Que J, Liu K, Wang H, Liao S: Netrin-1 Ameliorates Blood-Brain Barrier Impairment Secondary to Ischemic Stroke via the Activation of PI3K Pathway. Front Neurosci 2017, 11:700.

58. Cunningham CL, Martinez-Cerdeno V, Noctor SC: Microglia regulate the number of neural precursor cells in the developing cerebral cortex. J Neurosci 2013, 33:4216-4233.

59. Biber K, Neumann H, Inoue K, Boddeke HW: Neuronal 'On' and 'Off' signals control microglia. Trends Neurosci 2007, 30:596-602.

60. Hinkle JT, Dawson VL, Dawson TM: The A1 astrocyte paradigm: New avenues for pharmacological intervention in neurodegeneration. Mov Disord 2019, 34:959-969.

61. Li T, Chen X, Zhang C, Zhang Y, Yao W: An update on reactive astrocytes in chronic pain. J Neuroinflammation 2019, 16:140.

62. Yavas E, Gonzalez S, Fanselow MS: Interactions between the hippocampus, prefrontal cortex, and amygdala support complex learning and memory. F1000Res 2019, 8. 
63. Dolleman-van der Weel MJ, Griffin AL, Ito HT, Shapiro ML, Witter MP, Vertes RP, Allen TA: The nucleus reuniens of the thalamus sits at the nexus of a hippocampus and medial prefrontal cortex circuit enabling memory and behavior. Learn Mem 2019, 26:191-205.

64. Serhan CN, de la Rosa X, Jouvene C: Novel mediators and mechanisms in the resolution of infectious inflammation: evidence for vagus regulation. J Intern Med 2019, 286:240-258.

65. Moore SW, Lai Wing Sun K, Xie F, Barker PA, Conti M, Kennedy TE: Soluble adenylyl cyclase is not required for axon guidance to netrin-1. J Neurosci 2008, 28:3920-3924.

66. Wu KY, Zippin JH, Huron DR, Kamenetsky M, Hengst U, Buck J, Levin LR, Jaffrey SR: Soluble adenylyl cyclase is required for netrin-1 signaling in nerve growth cones. Nat Neurosci 2006, 9:1257-1264.

67. Ranganathan PV, Jayakumar C, Mohamed R, Dong Z, Ramesh G: Netrin-1 regulates the inflammatory response of neutrophils and macrophages, and suppresses ischemic acute kidney injury by inhibiting COX-2-mediated PGE2 production. Kidney Int 2013, 83:1087-1098.

\section{Figures}


A

$6 h$

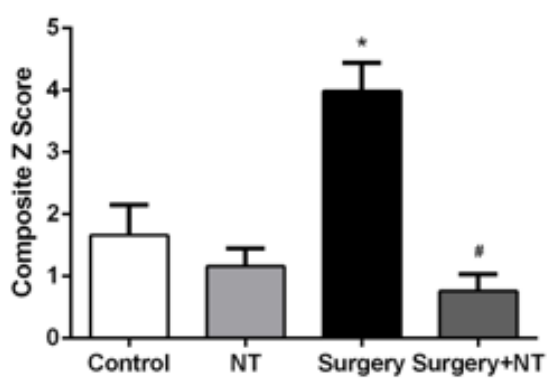

D

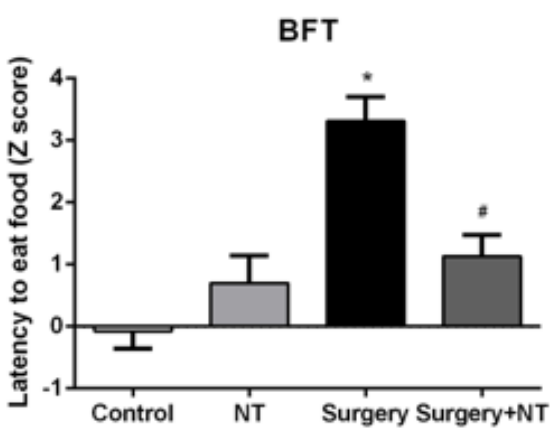

G

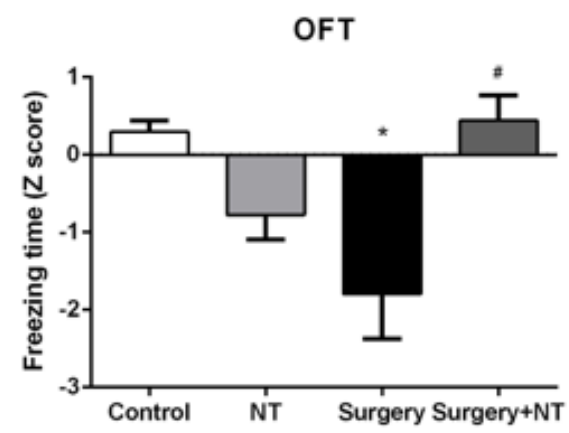

B

9h

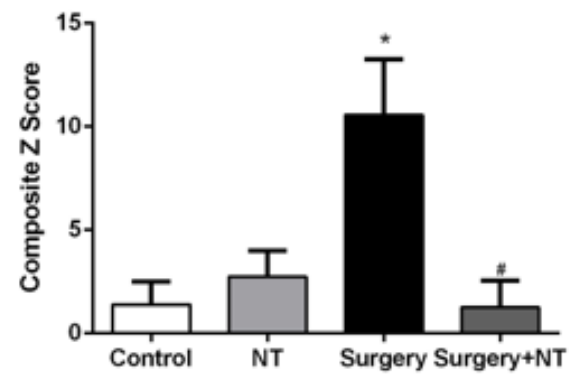

E

OFT

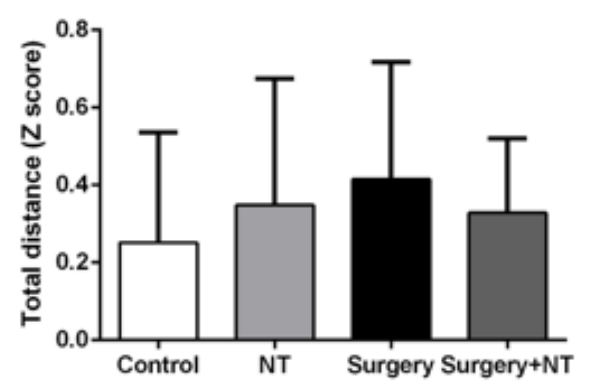

п

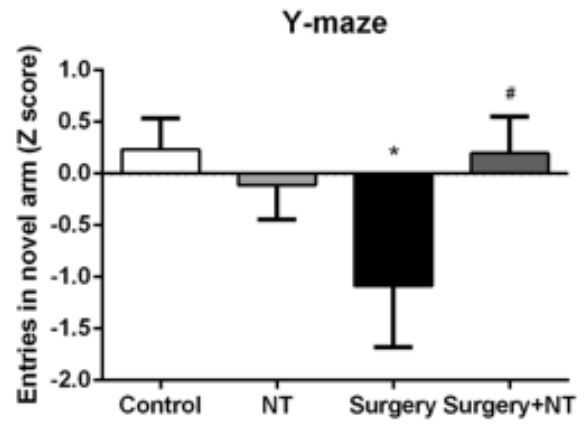

C

24h

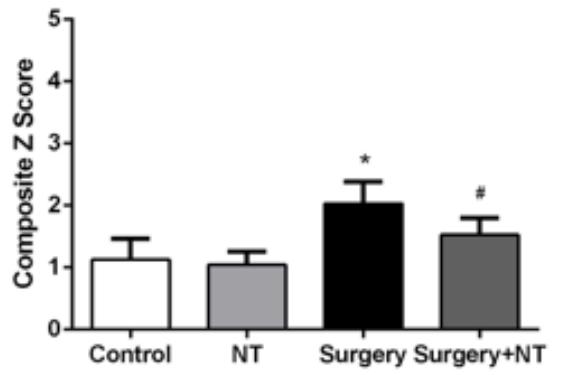

F
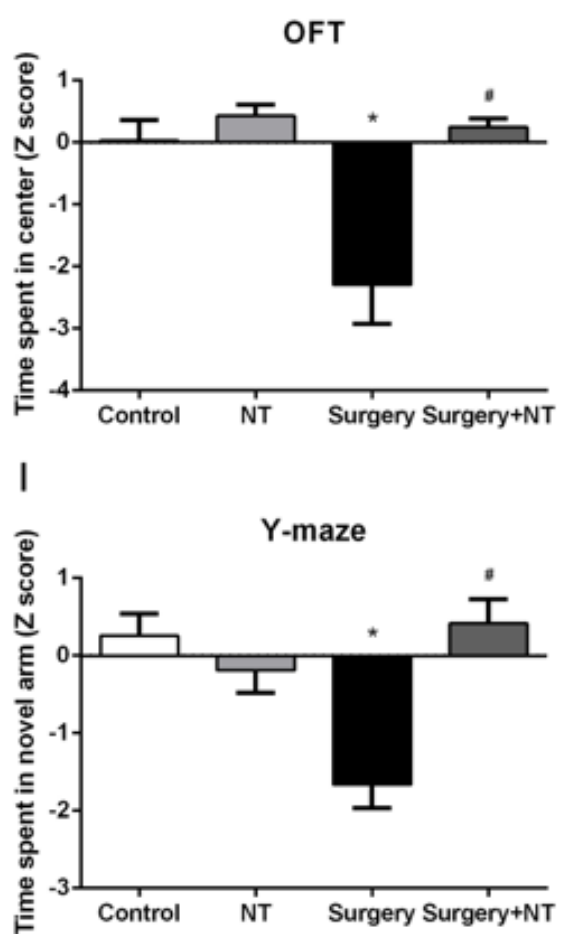

Figure 1

Exogenous recombinant human Netrin-1 (rh-NTN-1) improved POD-like behavior on surgical mice. (A-C) The changes of composite Z scores after rh-NTN-1 treatments via tail vein injection at 6,9,24 hours postoperatively. (D) Surgery/Anesthesia increased the time of latency to eat food while exogenous rhNTN-1 significantly decreased it. (E) Surgery/Anesthesia has no influence in total distance. (F-I) Surgery/Anesthesia decreased z scores at 9 hours postoperatively, while exogenous rh-NTN-1 significantly increased $z$ scores. The data are presented as the means \pm standard error of the mean for each group ( $n=10$ per cohort). ${ }^{\star} P<0.05$ versus the control group, $\# P<0.05$ versus the surgery group. 
A

$6 h$

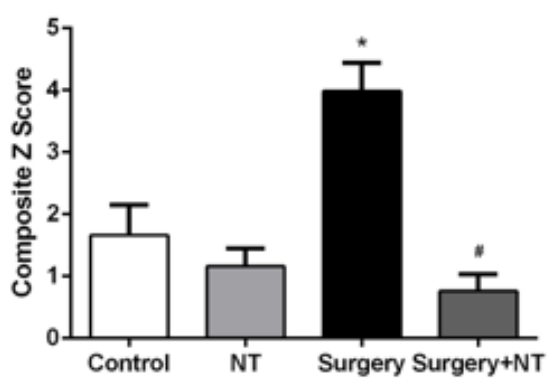

D

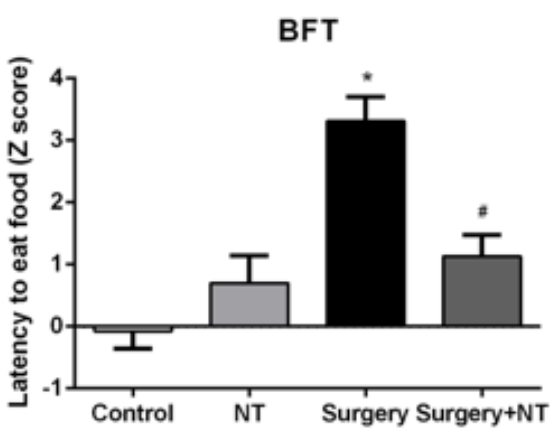

G

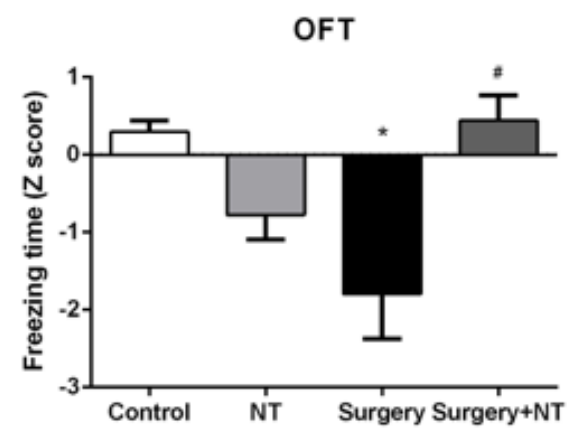

B

9h

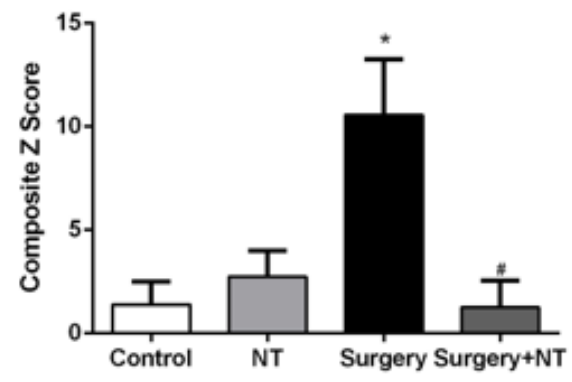

E

OFT

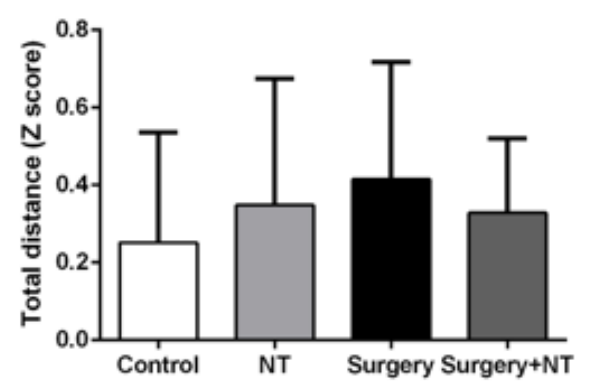

п

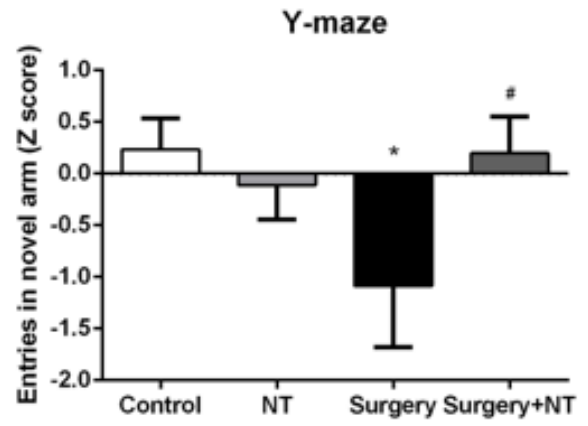

C

24h

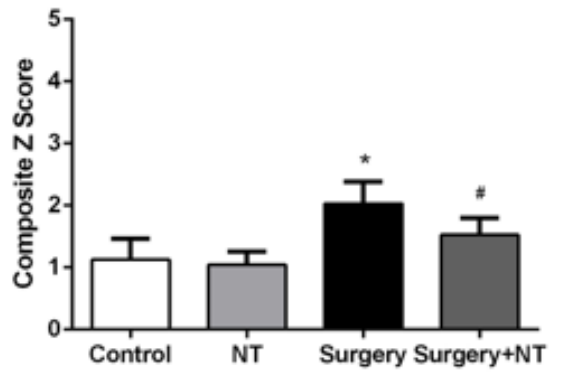

F
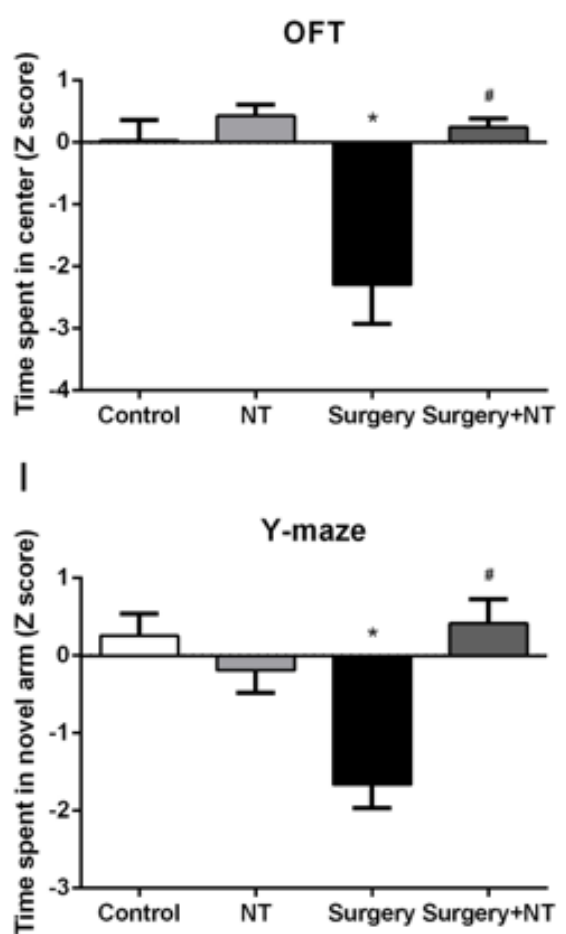

Figure 1

Exogenous recombinant human Netrin-1 (rh-NTN-1) improved POD-like behavior on surgical mice. (A-C) The changes of composite Z scores after rh-NTN-1 treatments via tail vein injection at 6,9,24 hours postoperatively. (D) Surgery/Anesthesia increased the time of latency to eat food while exogenous rhNTN-1 significantly decreased it. (E) Surgery/Anesthesia has no influence in total distance. (F-I) Surgery/Anesthesia decreased z scores at 9 hours postoperatively, while exogenous rh-NTN-1 significantly increased $z$ scores. The data are presented as the means \pm standard error of the mean for each group ( $n=10$ per cohort). ${ }^{\star} P<0.05$ versus the control group, $\# P<0.05$ versus the surgery group. 
A

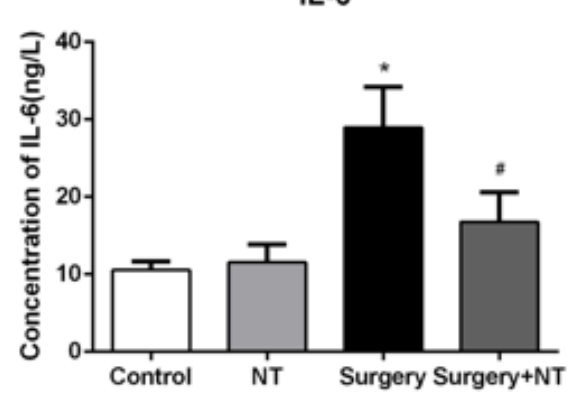

B

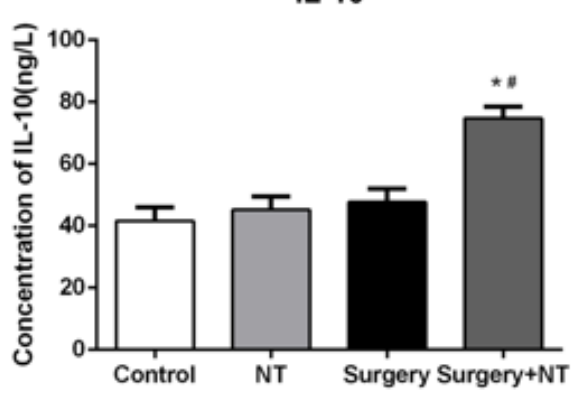

HMGB-1

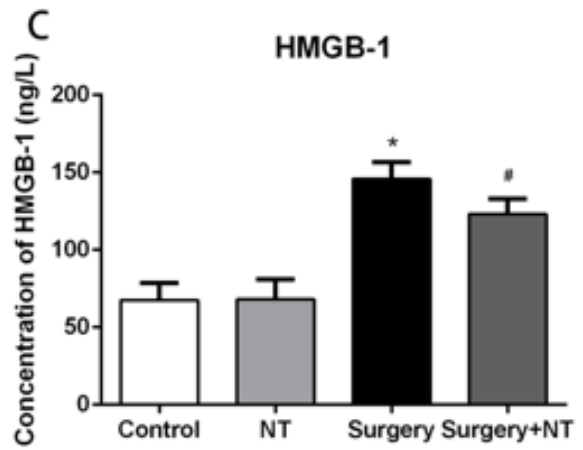

Figure 2

Exogenous recombinant human Netrin-1 (rh-NTN-1) decreased the levels of proinflammatory cytokines and increased the levels of anti-inflammatory cytokines in serum on surgical mice. (A-C) The expression levels of IL-6, IL-10 and HMGB-1 in serum at 6 hours after surgery/anesthesia. The data are presented as the means \pm standard error of the mean for each group ( $n=5$ per cohort). ${ }^{*}<0.05$ versus the control group, $\# \mathrm{P}<0.05$ versus the surgery group.

A

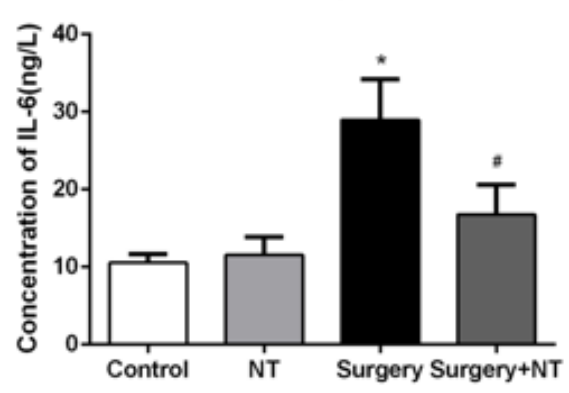

B

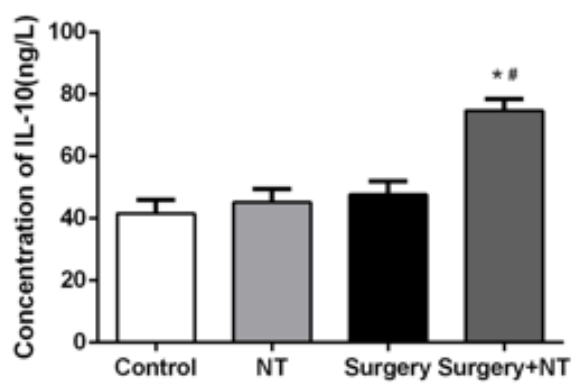

HMGB-1

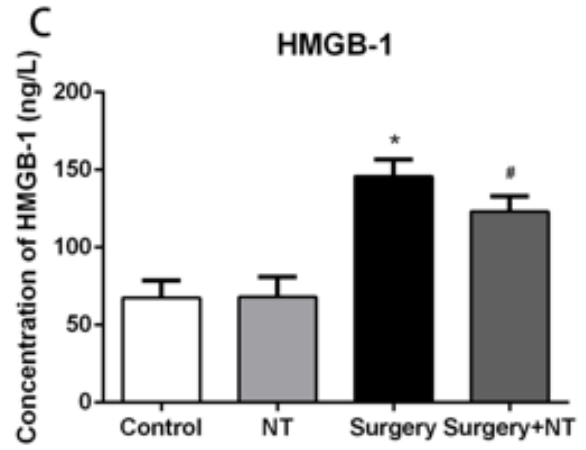

Figure 2

Exogenous recombinant human Netrin-1 (rh-NTN-1) decreased the levels of proinflammatory cytokines and increased the levels of anti-inflammatory cytokines in serum on surgical mice. (A-C) The expression levels of IL-6, IL-10 and HMGB-1 in serum at 6 hours after surgery/anesthesia. The data are presented as the means \pm standard error of the mean for each group ( $n=5$ per cohort). ${ }^{*} P<0.05$ versus the control group, $\# \mathrm{P}<0.05$ versus the surgery group. 
A

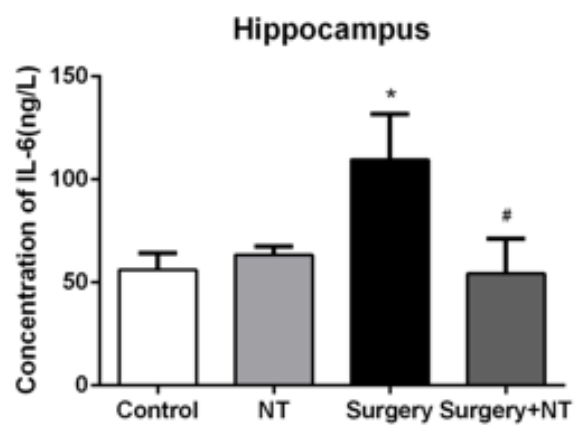

D

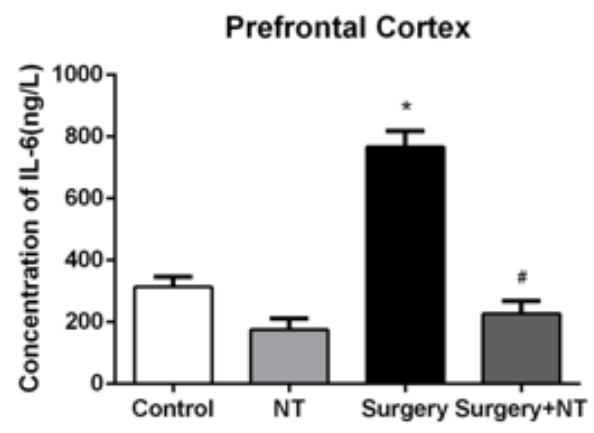

B

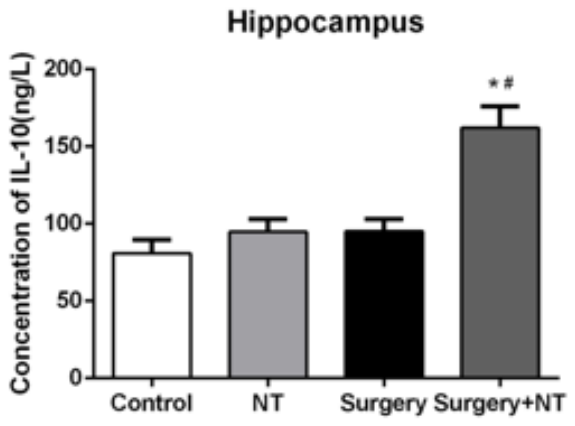

$\mathrm{E}$

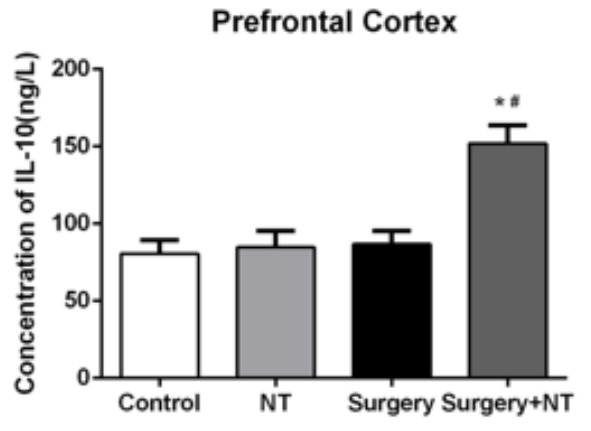

C

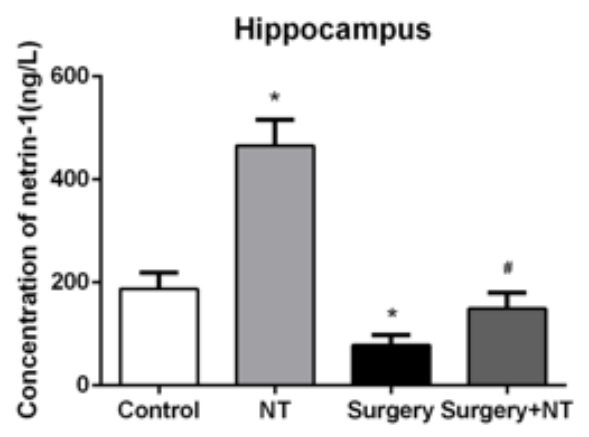

F

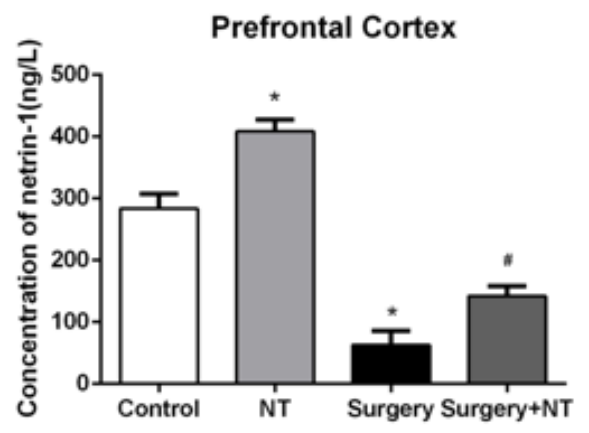

Figure 3

Exogenous recombinant human Netrin-1 (rh-NTN-1) decreased the levels of proinflammatory cytokines, increased the levels of anti-inflammatory cytokines and netrin-1 in prefrontal cortex and hippocampus of surgical mice. (A-C) ELISA was used to detect the expression of IL-6, IL-10 and netrin-1 in hippocampus at 6 hours postoperatively. (D-F) The altered expression of IL-6, IL-10 and netrin- 1 in prefrontal cortex at 6 hours postoperatively. The data are presented as the means \pm standard error of the mean for each group ( $n=5$ per cohort). ${ }^{*}<<0.05$ versus the control group, $\# P<0.05$ versus the surgery group. 
A

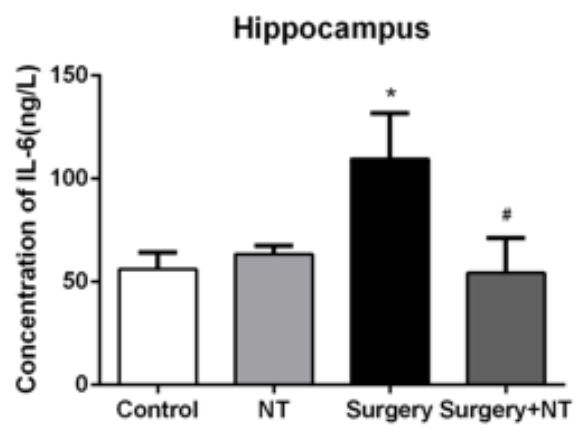

D

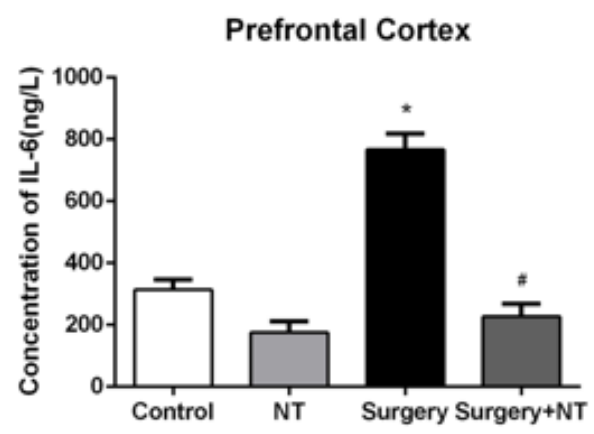

B

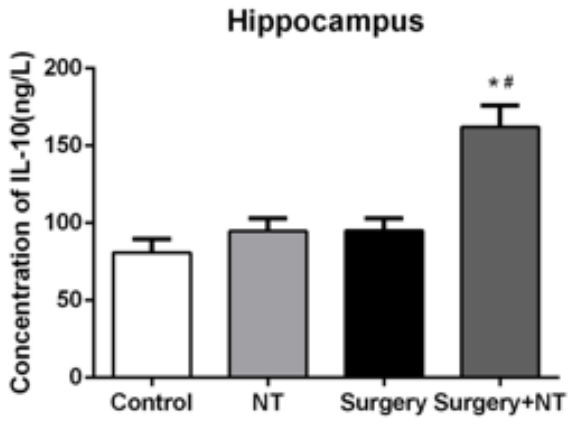

$\mathrm{E}$

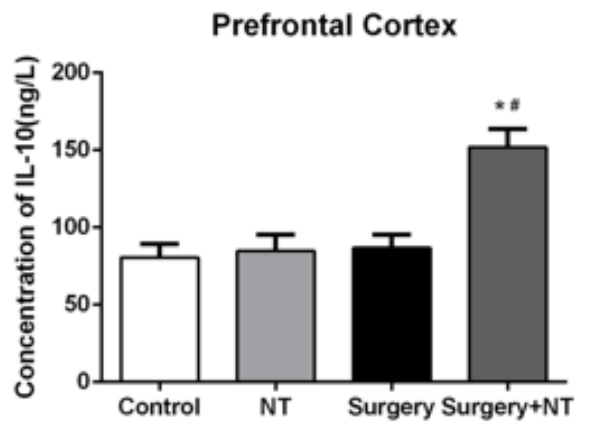

C

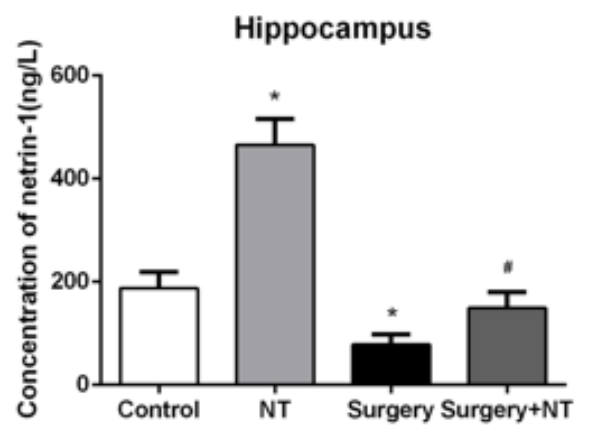

$\mathrm{F}$

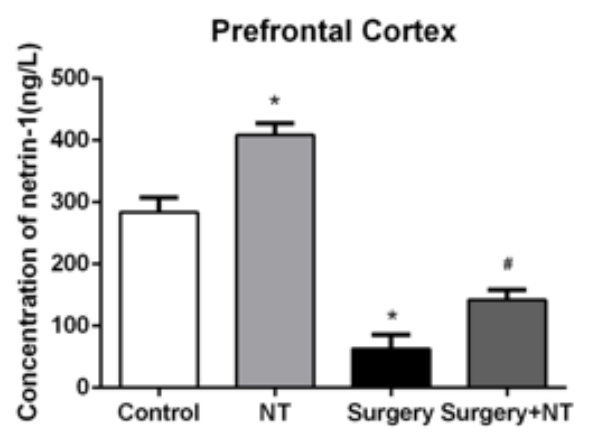

Figure 3

Exogenous recombinant human Netrin-1 (rh-NTN-1) decreased the levels of proinflammatory cytokines, increased the levels of anti-inflammatory cytokines and netrin-1 in prefrontal cortex and hippocampus of surgical mice. (A-C) ELISA was used to detect the expression of IL-6, IL-10 and netrin-1 in hippocampus at 6 hours postoperatively. (D-F) The altered expression of IL-6, IL-10 and netrin-1 in prefrontal cortex at 6 hours postoperatively. The data are presented as the means \pm standard error of the mean for each group ( $n=5$ per cohort). ${ }^{*}<<0.05$ versus the control group, $\# P<0.05$ versus the surgery group. 

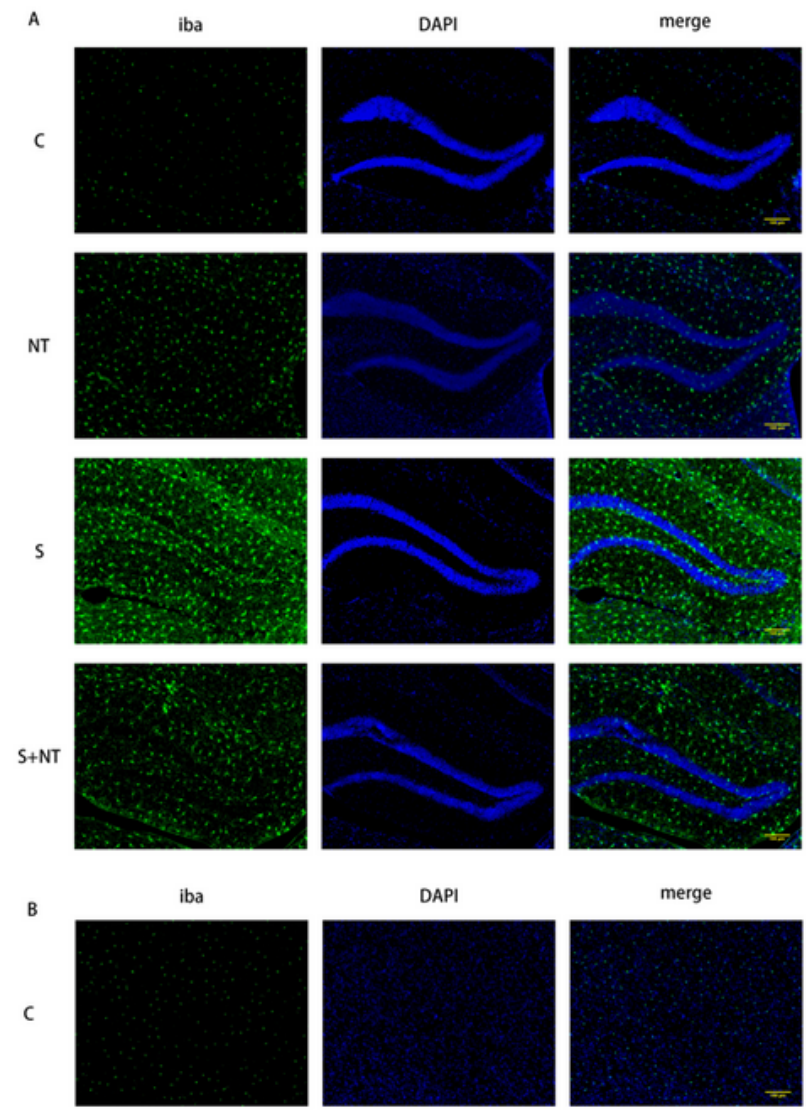

DAPI

merge

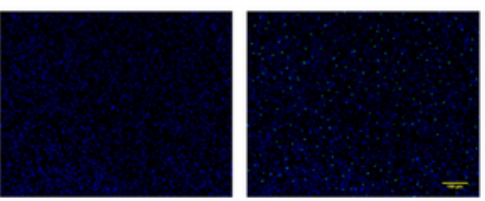

NT
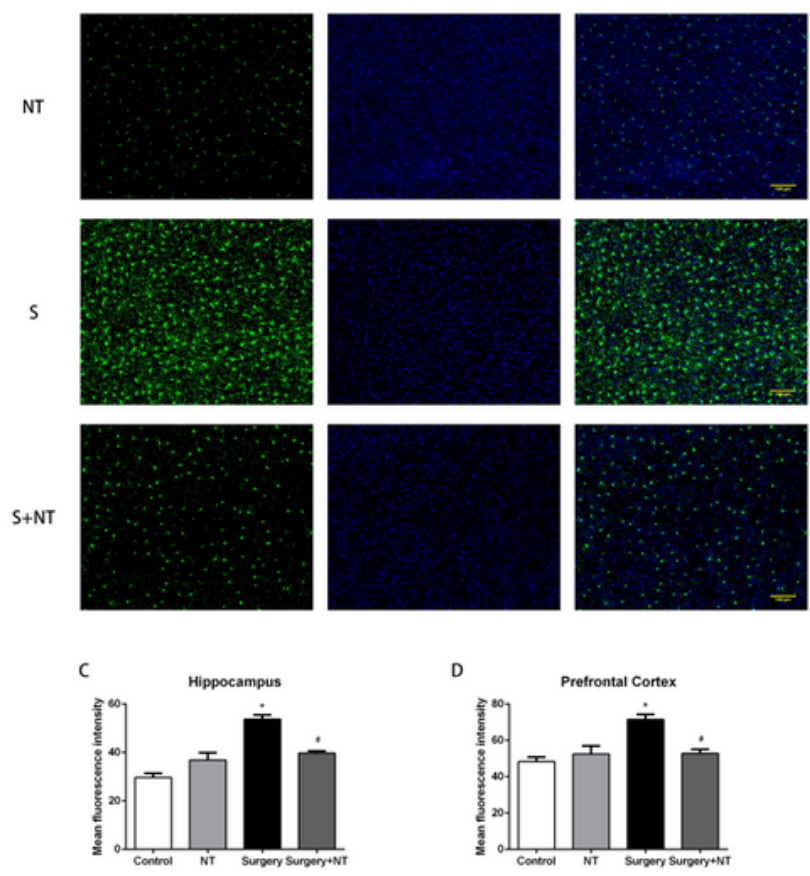

Figure 4

Exogenous recombinant human Netrin-1 (rh-NTN-1) decreased the activation of microglia on surgical mice. Activation of microglia in hippocampus (A) and prefrontal cortex (B) after 24 hours postoperatively. (C, D) Mean fluorescence intensity in hippocampus and prefrontal cortex after 24 hours postoperatively. The data are presented as the means \pm standard error of the mean for each group ( $n=3$ per cohort). ${ }^{*} \mathrm{P}<0.05$ versus the control group, $\# \mathrm{P}<0.05$ versus the surgery group. 

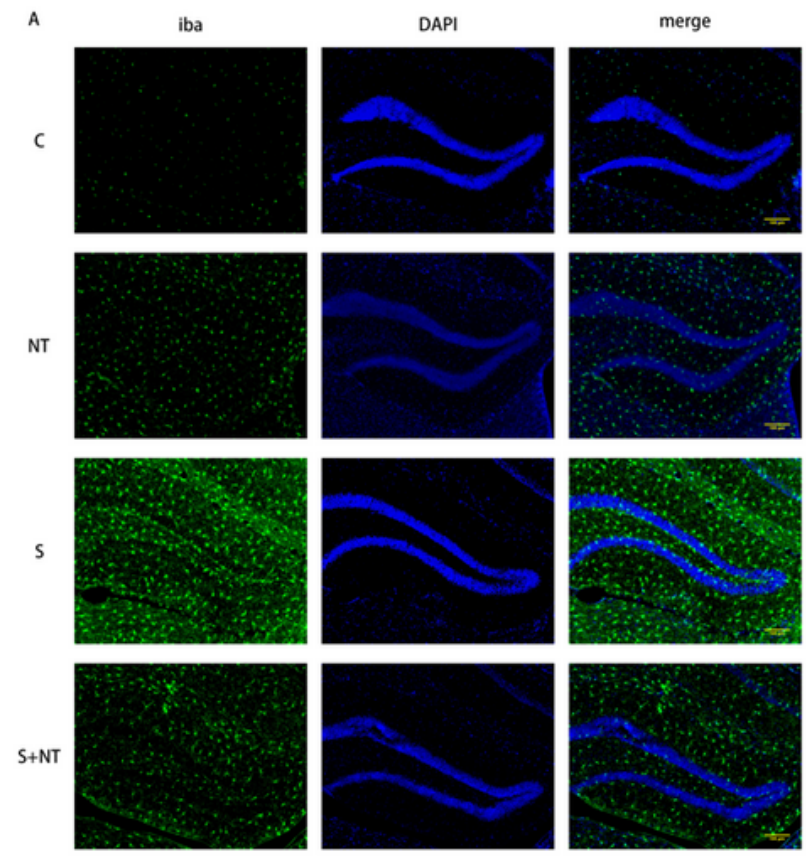

B

iba
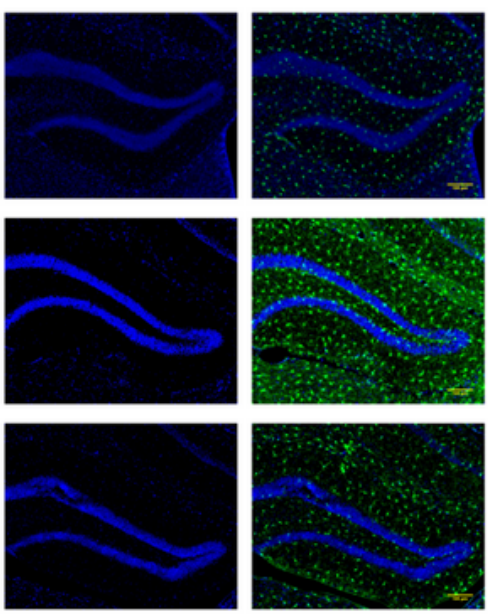

DAPI

merge

c
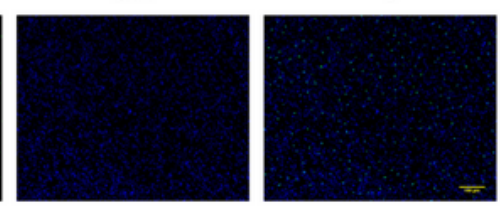

NT
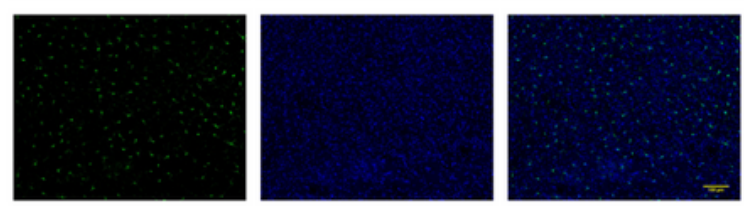

s
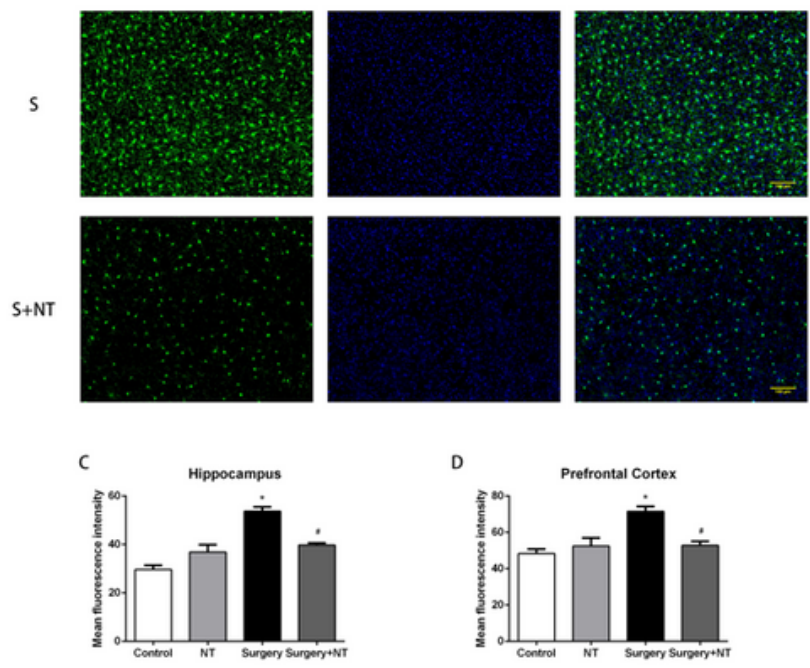

Figure 4

Exogenous recombinant human Netrin-1 (rh-NTN-1) decreased the activation of microglia on surgical mice. Activation of microglia in hippocampus (A) and prefrontal cortex (B) after 24 hours postoperatively. (C, D) Mean fluorescence intensity in hippocampus and prefrontal cortex after 24 hours postoperatively. The data are presented as the means \pm standard error of the mean for each group ( $n=3$ per cohort). ${ }^{*} \mathrm{P}<0.05$ versus the control group, $\# \mathrm{P}<0.05$ versus the surgery group. 


\section{Figure 5}

Exogenous recombinant human Netrin-1 (rh-NTN-1) attenuates the anesthesia/surgery -induced increase in blood-brain barrier permeability of dextran in the hippocampus of mice. $\triangle A \mathbb{A}$ Immunostaining of blood vessels (lectin, green, column a) and dextran (10-kDa dextran, red, column b) of the brain section inthe four groups. The red spots (non-overlap area) in column cindicate the dextran that is not inside the blood vessel (extravascular dextran). $\mathrm{N}=$ total of 150 slides from 5 mice in each group. (B) Spectrophotometric quantification of brain dextran (10 kDa) level. The anesthesia/ surgery increases brain dextran level as compared to that in the control condition of mice. Treatment with rh-NTN-1 attenuates the anesthesia/surgery-induced increase in the extravascular dextran level in the mice. The data are presented as the means \pm standard error of the mean for each group ( $n=5$ per cohort). ${ }^{*}<<0.05$ versus the control group. 
A

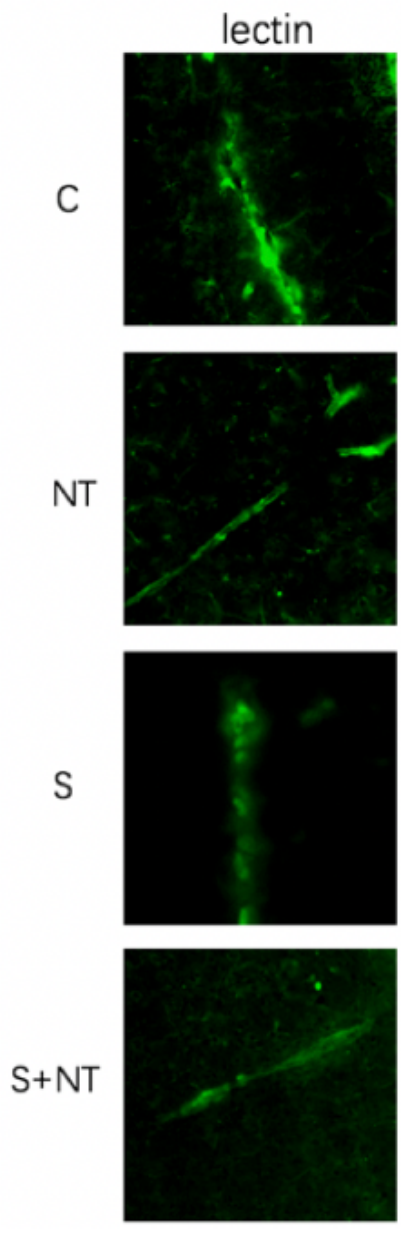

(a)
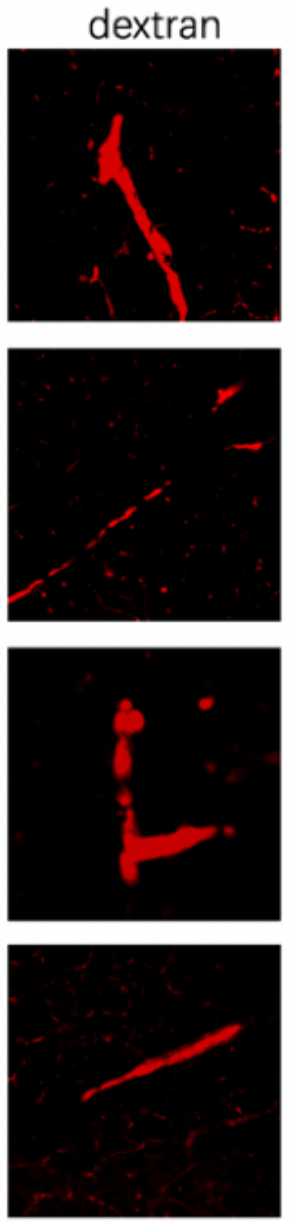

(b)
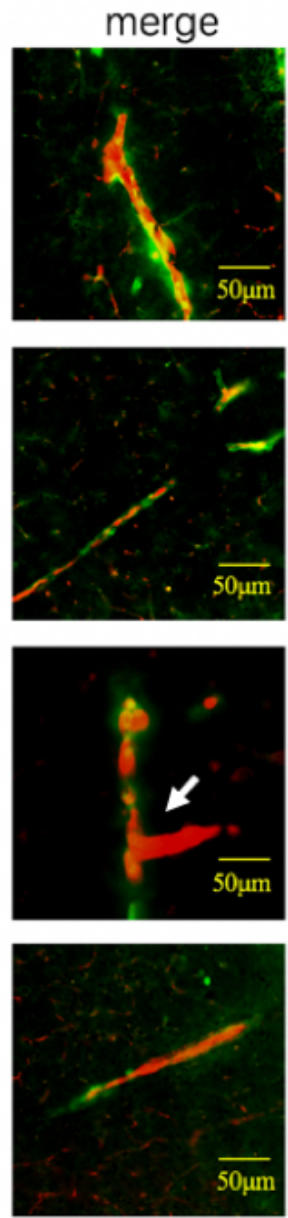

(c)

B Spectrophotometric Quantification of Dextran

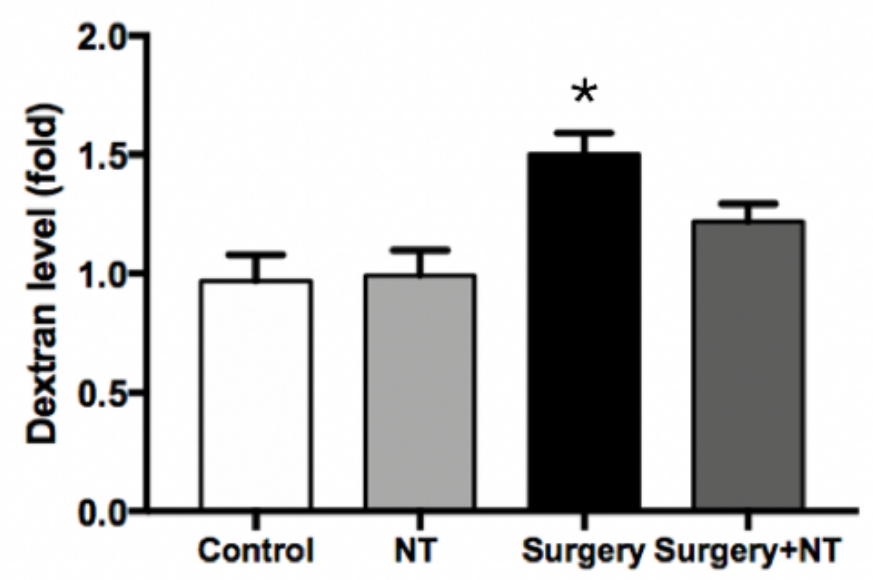

Figure 5

Exogenous recombinant human Netrin-1 (rh-NTN-1) attenuates the anesthesia/surgery -induced increase

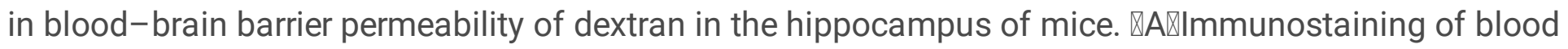
vessels (lectin, green, column a) and dextran (10-kDa dextran, red, column b) of the brain section inthe four groups. The red spots (non-overlap area) in column cindicate the dextran that is not inside the blood vessel (extravascular dextran). $\mathrm{N}$ = total of 150 slides from 5 mice in each group. (B) Spectrophotometric 
quantification of brain dextran (10 kDa) level. The anesthesia/ surgery increases brain dextran level as compared to that in the control condition of mice. Treatment with rh-NTN-1 attenuates the anesthesia/surgery-induced increase in the extravascular dextran level in the mice. The data are presented as the means \pm standard error of the mean for each group ( $n=5$ per cohort). ${ }^{*} P<0.05$ versus the control group.

A

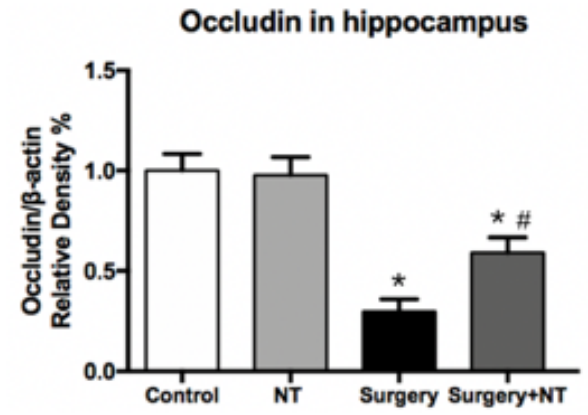

D

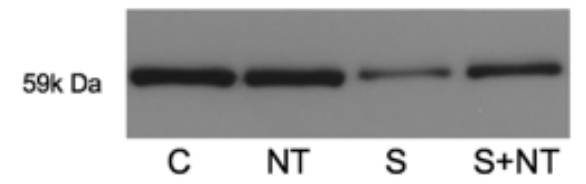

${ }^{43 \mathrm{k} \mathrm{Da}}$

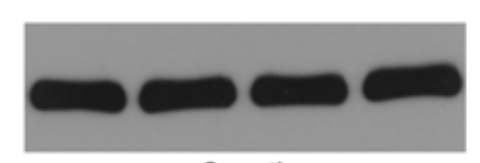

$\beta$-actin
B

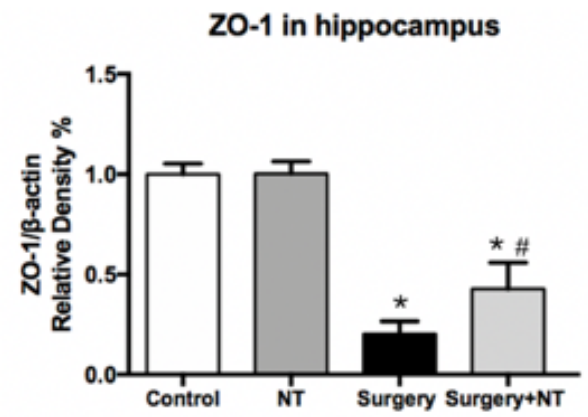

$\mathrm{E}$
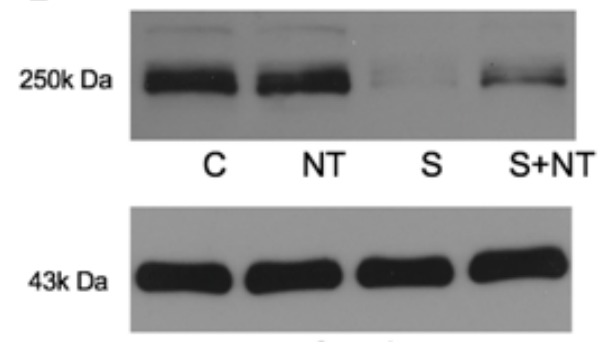

$\beta$-actin
C

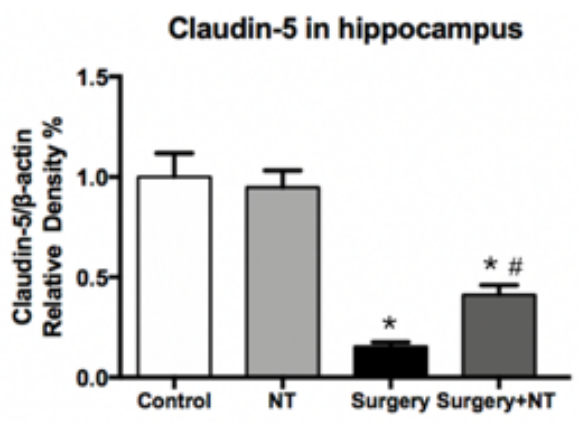

$\mathrm{F}$

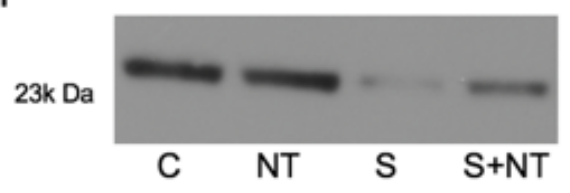

43k Da

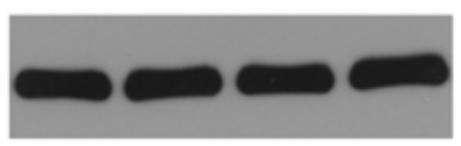

$\beta$-actin

Figure 6

Exogenous recombinant human Netrin-1 (rh-NTN-1) attenuates the anesthesia/ surgery-induced reduction in cell junction proteins in the hippocampus of mice. The anesthesia/ surgery reduces protein levels of occludin (A,D), ZO-1 (B,E)an claudin-5 (C,F) in the hippocampus of mice as compared to control condition at $9 \mathrm{~h}$ after the anesthesia/surgery. Treatment of rh-NTN-1 attenuates these reductions in the levels of occludin ( $A, D), Z O-1(B, E)$ an claudin-5 $(C, F)$. The data are presented as the means \pm standard error of the mean for each group $\left(n=5\right.$ per cohort). ${ }^{*}<0.05$ versus the control group, $\# P<0.05$ versus the surgery group.

\section{Figure 6}

Exogenous recombinant human Netrin-1 (rh-NTN-1) attenuates the anesthesia/ surgery-induced reduction in cell junction proteins in the hippocampus of mice. The anesthesia/ surgery reduces protein levels of occludin (A,D), ZO-1 (B,E)an claudin-5 (C,F) in the hippocampus of mice as compared to control condition at $9 \mathrm{~h}$ after the anesthesia/surgery. Treatment of rh-NTN-1 attenuates these reductions in the levels of occludin $(A, D), Z O-1(B, E)$ an claudin-5 $(C, F)$. The data are presented as the means \pm standard error of the 
mean for each group ( $\mathrm{n}=5$ per cohort). ${ }^{*} \mathrm{P}<0.05$ versus the control group, $\# \mathrm{P}<0.05$ versus the surgery group.

A

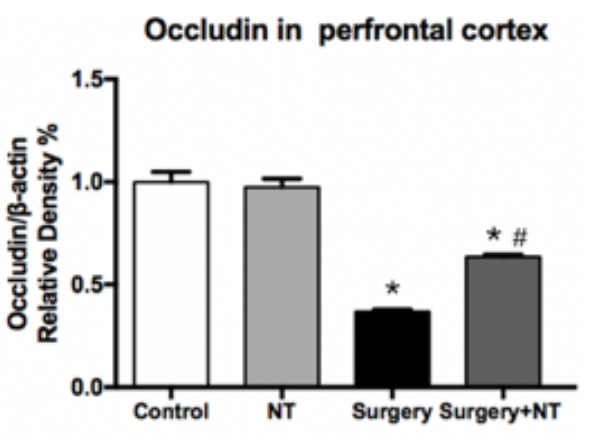

D

$59 \mathrm{k} \mathrm{Da}$

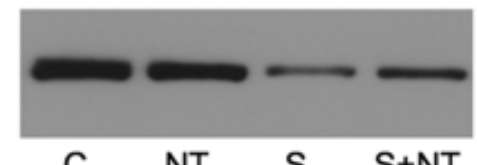

$43 \mathrm{k} \mathrm{Da}$

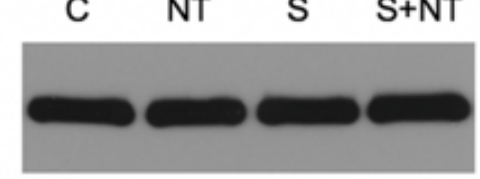

$\beta$-actin
B

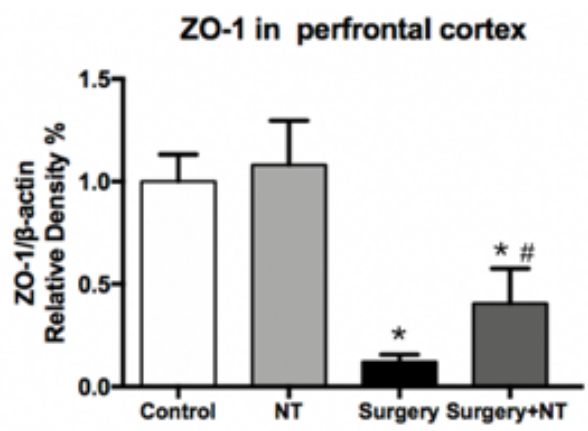

$\mathrm{E}$

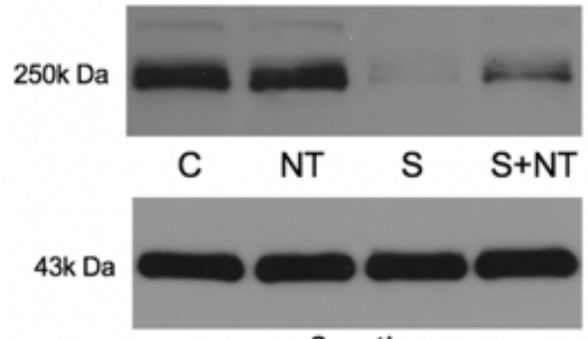

$\beta$-actin
C

Claudin-5 in perfrontal cortex

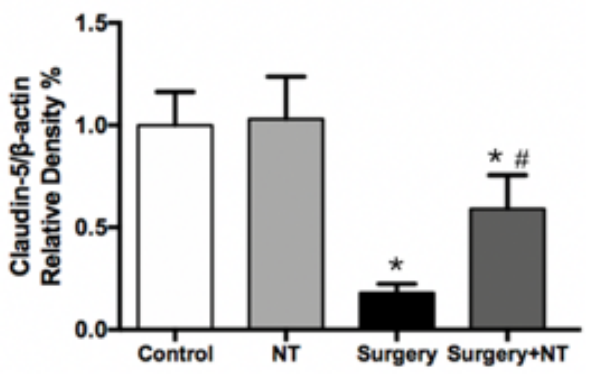

$\mathrm{F}$

23k Da

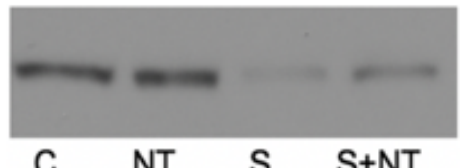

$43 \mathrm{k} \mathrm{Da}$

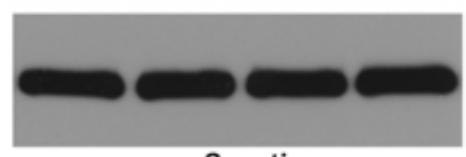

$\beta$-actin

Figure 7

Exogenous recombinant human Netrin-1 (rh-NTN-1) attenuates the anesthesia/surgery-induced reduction in cell junction proteins in the perfrontal cortex of mice. The anesthesia/surgery reduces protein levels of occludin (A,D), ZO-1 (B,E)an claudin-5 (C,F) in the perfrontal cortex of mice as compared to control condition at $9 \mathrm{~h}$ after the anesthesia/surgery. Treatment of rh-NTN-1 attenuates these reductions in the levels of occludin $(A, D), Z O-1(B, E)$ an claudin-5 $(C, F)$. The data are presented as the means \pm standard error of the mean for each group ( $n=5$ per cohort). ${ }^{*}<<0.05$ versus the control group, $\# P<0.05$ versus the surgery group. 
A

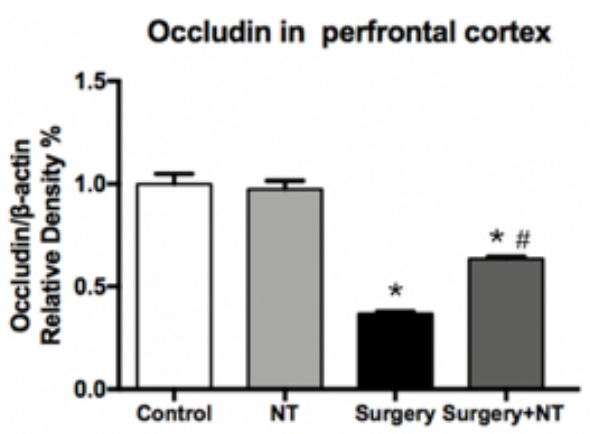

D

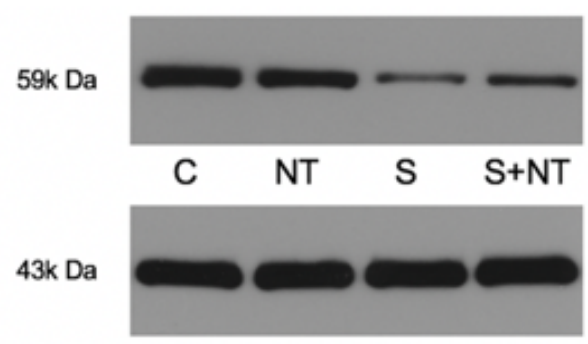

$\beta$-actin
B

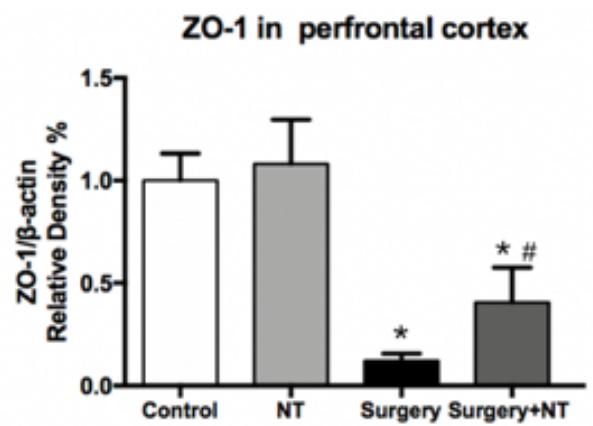

E

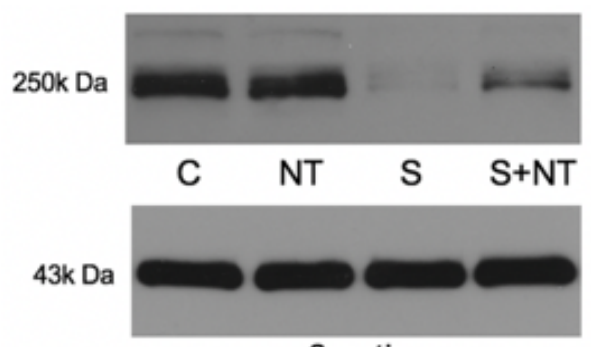

$\beta$-actin
C

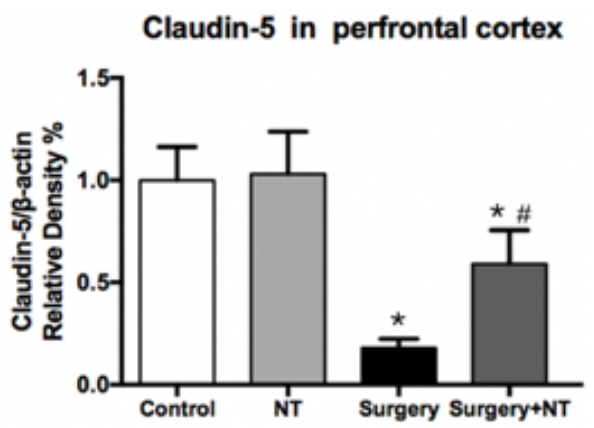

F

23k Da

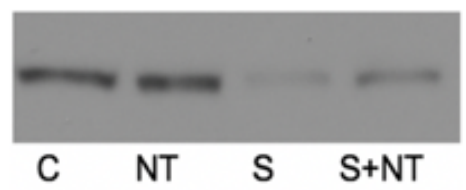

43k Da

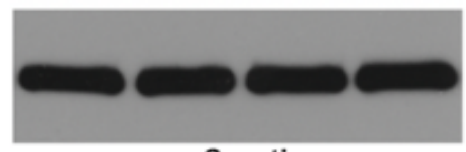

$\beta$-actin

Figure 7

Exogenous recombinant human Netrin-1 (rh-NTN-1) attenuates the anesthesia/surgery-induced reduction in cell junction proteins in the perfrontal cortex of mice. The anesthesia/surgery reduces protein levels of occludin (A,D), ZO-1 (B,E)an claudin-5 (C,F) in the perfrontal cortex of mice as compared to control condition at $9 \mathrm{~h}$ after the anesthesia/surgery. Treatment of rh-NTN-1 attenuates these reductions in the levels of occludin (A,D), ZO-1 (B,E)an claudin-5 (C,F). The data are presented as the means \pm standard error of the mean for each group ( $n=5$ per cohort). ${ }^{*}<<0.05$ versus the control group, $\# P<0.05$ versus the surgery group. 